Survey Paper

\title{
A survey on probabilistic broadcast schemes for wireless ad hoc networks
}

\author{
D.G. Reina ${ }^{a}$, S.L. Toral ${ }^{\text {a,* }}$, P. Johnson ${ }^{\text {b }}$, F. Barrero ${ }^{a}$ \\ ${ }^{a}$ University of Seville, Escuela Superior de Ingenieros, Avda. Camino de los Descubrimientos, s/n, 41092 Sevilla, Spain \\ ${ }^{\mathrm{b}}$ Liverpool John Moores University, Liverpool, UK
}

\section{A R T I C L E I N F O}

\section{Article history:}

Received 7 March 2014

Received in revised form 22 July 2014

Accepted 2 October 2014

Available online $\mathrm{xxxx}$

\section{Keywords:}

Probabilistic broadcast

Flooding

Routing protocols

Wireless ad hoc networks

\begin{abstract}
A B S T R A C T
Broadcast or flooding is a dissemination technique of paramount importance in wireless ad hoc networks. The broadcast scheme is widely used within routing protocols by a wide range of wireless ad hoc networks such as mobile ad hoc networks, vehicular ad hoc networks, and wireless sensor networks, and used to spread emergency messages in critical scenarios after a disaster scenario and/or an accidents. As the type broadcast scheme used plays an important role in the performance of the network, it has to be selected carefully. Though several types of broadcast schemes have been proposed, probabilistic broadcast schemes have been demonstrated to be suitable schemes for wireless ad hoc networks due to a range of benefits offered by them such as low overhead, balanced energy consumption, and robustness against failures and mobility of nodes. In the last decade, many probabilistic broadcast schemes have been proposed by researchers. In addition to reviewing the main features of the probabilistic schemes found in the literature, we also present a classification of the probabilistic schemes, an exhaustive review of the evaluation methodology including their performance metrics, types of network simulators, their comparisons, and present some examples of real implementations, in this paper.
\end{abstract}

(c) 2014 Elsevier B.V. All rights reserved.

\section{Introduction}

Broadcasting is a widely used dissemination technique in which nodes send out the same information simultaneously to all their neighbors. Broadcasting is used in ad hoc networks such as Wireless Sensor Networks (WSNs) [7,121,106], Mobile Ad Hoc Networks (MANETs) [48], and Vehicular Ad Hoc Networks (VANETs) [107,116]. In routing protocols for ad hoc networks, broadcasting is part of the discovery phase, which is responsible for finding a communication path to route the application data from a source node to one or more destination nodes (unicast [18] or multicast routing protocols $[6,5,26])$. Broadcasting is also used in the maintenance of routes since nodes exchange

* Corresponding author.
Hello packets to collect neighboring information. In addition, broadcasting is also employed to disseminate emergency or warning messages in disaster scenarios, which is one of the main applications of MANETs [96,95,92]. In such harsh conditions, an efficient broadcast mechanism is vital and may be the only possible way to disseminate crucial information [40,97]. Furthermore, in VANETs the dissemination of warning messages is also important to warn the motorists of congestions due to traffic accidents.

The simplest way of broadcasting is flooding, in which each node in the network retransmits an incoming message once. The main benefit of using flooding is the ease of implementation. However, this technique also has several issues, one of them being its inefficiency in terms of resource consumption such as bandwidth and energy. While the former impacts the network in terms of efficiency and capacity, the latter is crucial for the lifetime 
of the network. The main problem of flooding in wireless ad hoc networks is that it causes the well-known broadcast storm problem [109] due to collisions and contention. Since all the nodes share the same wireless medium, the broadcast storm problem leads to dramatic deterioration of the performance in wireless ad hoc networks. In order to alleviate the broadcast storm problem many broadcast protocols have been proposed in the last decade [31]. A basic classification of broadcast schemes divides them into two categories, deterministic schemes and probabilistic schemes. In deterministic techniques, only a subset of nodes are allowed to take part in the broadcasting process. Multi Point Relay (MPR) [65] and Connected Dominating Set (CDS) are some examples of deterministic broadcast algorithms. However, this could lead to repeated use of the same nodes. In addition, under mobility conditions this set of nodes should change very frequently because of the topological changes. Probabilistic broadcast schemes however balance the power consumption among all the nodes in the network by selecting well balanced routes over the network lifetime. In probabilistic broadcast, nodes forward the incoming broadcast packets according to certain probability value, so all nodes are allowed to participate in the broadcast process. Moreover, probabilistic schemes are more robust against failures, attacks, and are unaffected by the mobility of nodes like the deterministic schemes.

This paper surveys the main probabilistic schemes proposed in the literature for wireless ad hoc networks. As fast as we know, this is the first survey of probabilistic broadcast in ad hoc networks. The main contributions of this paper are:

- A classification of probabilistic broadcast schemes in wireless ad hoc networks.

- A thorough review of the proposed schemes found in the literature.

- A review of the evaluation methodology for probabilistic broadcast, including performance metrics, simulation platforms, comparisons and real implementations.

- To provide a set of open challenges of probabilistic broadcast schemes that have not been covered yet.
This survey continues as follows, the proposed classification of probabilistic broadcast schemes is presented in Sections 2, and 3 reviews all the probabilistic schemes found in the literature that fall into the proposed classification. The methodology used to evaluate and compare the probabilistic schemes is reviewed in Section 4. Section 5 presents a discussion on the main findings of the proposed survey and provide a set of open challenges that have not been sufficiently covered in the literature so far. Finally, Section 6 includes the main conclusions of this survey.

\section{Classification of probabilistic broadcast schemes in wireless ad hoc networks}

The objective of this section is to classify the probabilistic broadcast schemes found in the literature. We have classified the probabilistic broadcast schemes into two main categories, (1) fixed probability schemes and (2) adaptive schemes (see Fig. 1). These main categories can be further divided into different subcategories as shown in Fig. 2 and explained below.

\subsection{Fixed probability schemes}

These schemes use a constant forwarding probability value so every node has the same forwarding probability in the network. Fixed probabilistic schemes have been studied using percolation theory and the phase transition phenomenon used in random networks [102,101,42,104]. However, there are important differences from random networks and ad hoc networks so the results observed in random networks cannot be said to be true for ad hoc networks. Consequently, there is not an optimal forwarding probability for all possible scenarios. This optimal forwarding probability may depend on many parameters such as density, distance among nodes, and speed.

\subsection{Adaptive schemes}

In adaptive schemes local or global parameters, such as density metrics, speed, energy are used to determine the

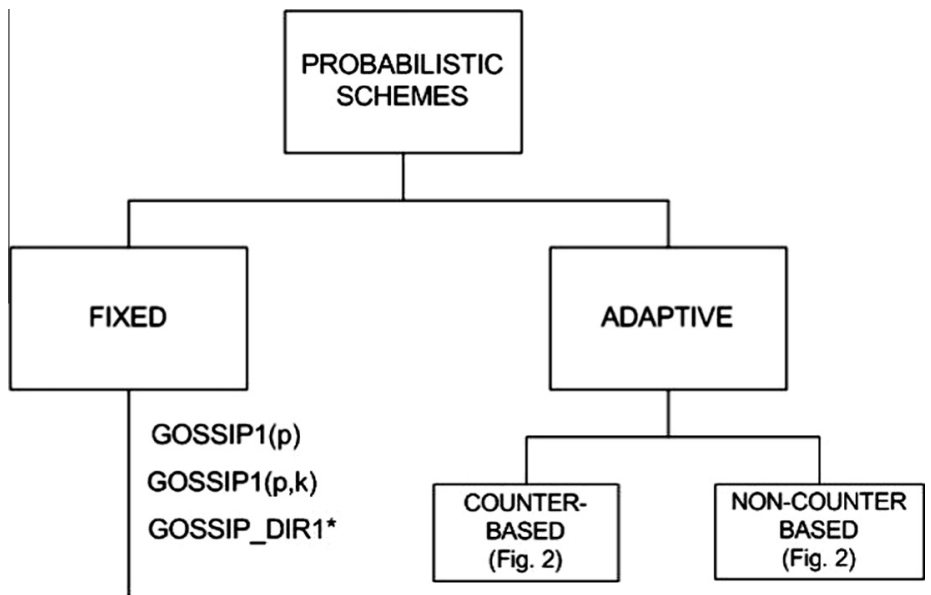

Fig. 1. Classification of probabilistic schemes. 


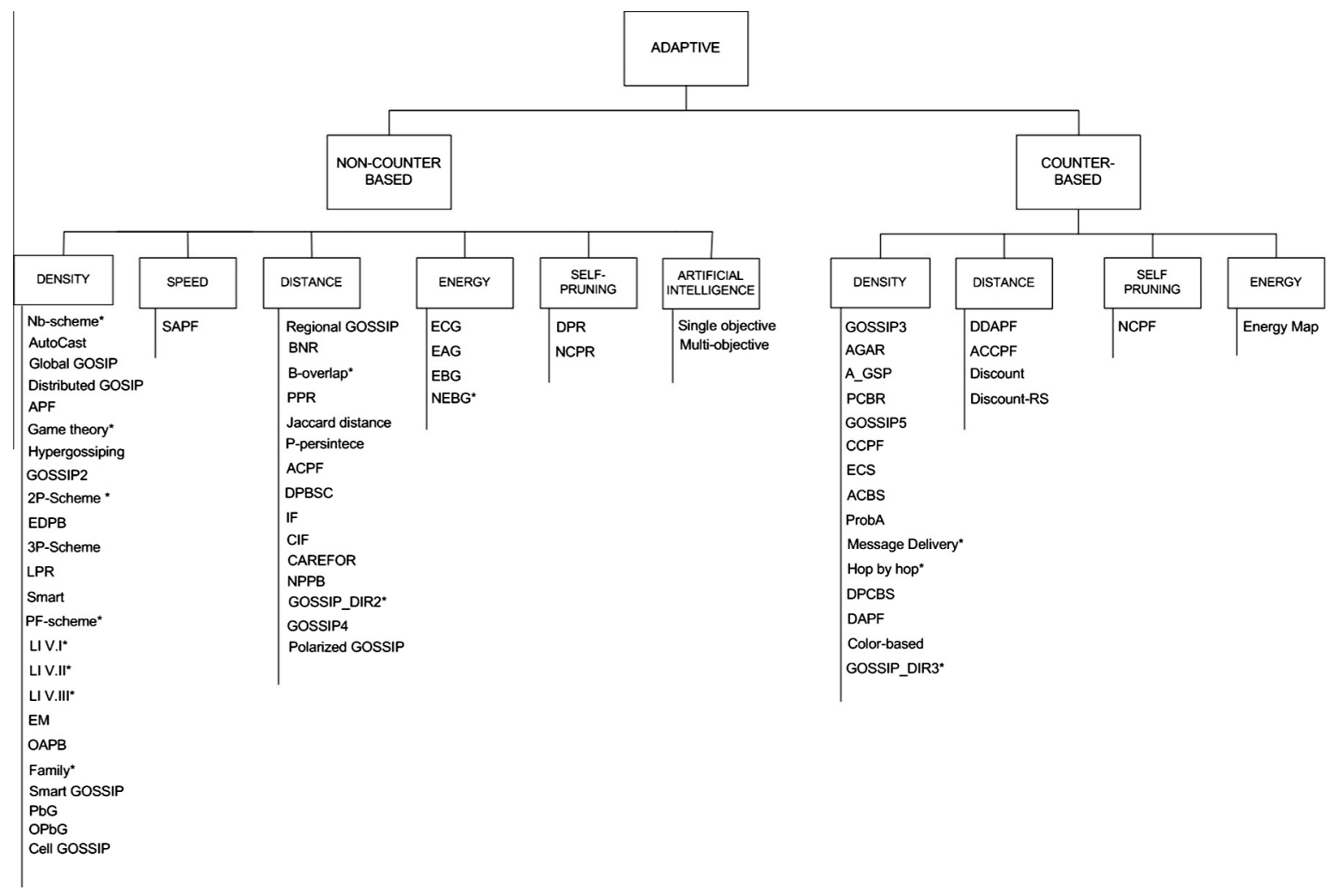

Fig. 2. Classification of adaptive schemes.

forwarding probability. The adaptive schemes can also be classified into two categories, (1) non-counter-based schemes and (2) counter-based schemes (see Fig. 2). The main difference between these two categories is that the counter-based schemes use the number of copies of a given broadcast packet as a feedback from the broadcast process in the node's neighborhood. The objective of the counterbased mechanism is to avoid the die out problem of broadcasting. The die out problem happens when the broadcast process is stopped due to forwarding decisions. Both counter-based and non counter-based adaptive schemes can be further classified according to the parameters used to adjust the forwarding probability such as density, distance, speed, number of neighbors covered (self-pruning), energy and artificial intelligence (see Fig. 2).

- Density-based schemes: These schemes use density metrics to adjust the forwarding probability. With regard to the density metrics used in adaptive schemes, these metrics range from local metrics such as a node's degree (number of neighbors) to global metrics such as the total number of nodes in the network. The main rationale behind density-based schemes is that the higher the density, the lower the forwarding probability.

- Distance-based schemes: These schemes use the distance among nodes as the main parameter to adjust the forwarding probability. The Euclidean distance is the most used type of distance, however, approximations based on the density of nodes, the Received
Signal Strength (RSS) and hop count are also employed. The basic idea is that nodes located further away from the senders are preferred since they avoid redundant retransmissions.

- Speed-based schemes: These schemes calculate the forwarding probability as a function of the node's speed. These schemes are intended for vehicular networks where the speed of the vehicles plays an important role.

- Self-pruning schemes: These schemes make the forwarding decision on the basis of the node's uncovered neighbors (self-pruning mechanism). The objective is to cover all of 2 hops neighbors of a node efficiently. Broadcast packets have to carry the sender's list of neighbors in order for the receiver to calculate the uncovered nodes.

- Energy-based schemes: These schemes use metrics related to the battery level of nodes to calculate the forwarding probability. These schemes are aimed at extending the network's lifetime. The idea is to favor nodes with more remaining energy.

- Artificial intelligence-based: These schemes use artificial intelligence to adjust the forwarding probability. Genetic algorithms are normally employed to adjust the forwarding probability.

\section{Review of existing probabilistic broadcast schemes}

This section reviews the main proposed probabilistic broadcast schemes found in the literature. It is organized 
Table 1

Notation.

\begin{tabular}{ll}
\hline Parameter & Description \\
\hline$d$ & Euclidean distance between two nodes \\
$h$ & Counter-based threshold \\
$m$ & Number of copies received of a given message \\
$N$ & Total number of nodes in the network \\
$n_{a v g}$ & Average number of neighbors of a given node \\
$n_{b}$ & Number of neighbors of a given node \\
$P_{r}$ & Power of a message received by a given node \\
$P_{t}$ & Power transmission of a given node \\
$p$ & Forwarding probability \\
$p_{i}$ & Initial forwarding probability used in schemes with \\
$r$ & different forwarding probabilities \\
$r$ & Node's radio transmission range \\
\hline
\end{tabular}

according to the classification presented in the previous Section 2, see Figs. 1 and 2. A summary table (Tables 2-10) has been included in each subsection. These tables present the main features of the reviewed schemes such as the name of proposed scheme (Figs. 1 and 2), the expression used to calculate the forwarding probability, the data that nodes need to collect to calculate the forwarding probability, the parameters that need to be adjusted, and the target application scenario of the broadcast schemes such as MANETs, VANETs and WSNs. Table 1 presents some notations widely used throughout this section.

\subsection{Fixed probability schemes}

As mentioned earlier, in fixed probability schemes, every node in the network forwards incoming broadcast packets with the same forwarding probability. The simplest probabilistic scheme is known as GOSSIP [42,41]). In this scheme, nodes forward an incoming packet with a fixed probability $p$, and the probability of not forwarding the incoming packet is $1-p$. This scheme is called as GOSSIP1(p). A further expansion is GOSSIP1(p,k) [42] in which nodes forward an incoming packet with a probability equal to 1 for the first $k$ hops. This ensures certain connectivity among nodes up to a distance of $k$ hops from the source node. The main problem of GOSSIP1 is determining the optimal forwarding probability for a given scenario. In [42], the authors demonstrated that the optimal probability is between $[0.65,0.75]$ in scenarios where the number of nodes is less than 1000. Moreover, the authors suggest that 0.5 could also be a good forwarding probability for a rectangle layout of $1650 \mathrm{~m} \times 600 \mathrm{~m}$ containing 150 nodes. Another important issue is how to fix the value of $k$. The authors used $k=4$ for large networks (more than 1000 nodes) with distances up to 45 hops. However, they used $k=1$ when they implemented GOSSIP over AODV [91] routing protocol in a more realistic scenario with 150 nodes. The results in [13] demonstrate that $p=0.5$ and $p=0.4$ can be optimum values for the forwarding probability to ensure reachability in networks with a number of nodes less than 100 (small to medium size networks). On the other hand, in [102] the authors evaluated GOSSIP1 with IEEE 802.11 MAC layer in DCF mode and high congestion conditions. The results show that a probability value as low as $p=0.1$ is sufficient to achieve high reachability in small to medium size network scenarios. A variant of GOSSIP1 is GOSSIP_DIR1 [104], which uses directional antennas instead of omnidirectional antennas. A summary of features of the probability schemes is shown in Table 2 .

\subsection{Adaptive probabilistic schemes}

Adaptive schemes tune the forwarding probability using any local or global parameter such as density and distance. As a general classification, we have classified adaptive schemes into two main categories: non counter-based schemes and counter-based schemes (see Fig. 2).

\subsubsection{Adaptive non-counter-based schemes}

As shown in Fig. 2, the non counter-based schemes can be divided into six groups based on density, distance, speed, self-pruning, energy and artificial intelligence schemes.

3.2.1.1. Density-based schemes. The density-based schemes can be further classified as shown in Fig. 3, where they are categorized into node's degree, density thresholds, and 2 hops information. The simplest parameter to tune the forwarding probability is the node's degree. The basic assumption is that the higher a node's degree, the lower its forwarding probability. An alternative is to take into account different density thresholds to adapt the forwarding probability. These thresholds are normally based on the node's degree. Moreover, in 2 hops information schemes, nodes should collect information from neighbor nodes (2 hops neighbors) and they adjust the forwarding probability according to the relationship between 1 hop and 2 hops neighbors.

(a) Node's degree or number of neighbors:

In $[32,33]$ (nb-scheme) the forwarding probability is adjusted to the inverse proportional of the number of neighbors of a node so $p=\frac{k}{n_{b}}$, where $k$ is the propagation factor and by adjusting its value, the maximum and minimum probability can be adjusted. The value of $n_{b}$ can be easily obtained via hello packets. Although this scheme is very simple, the optimum value of $k$ can depend on many topological parameters.

In AutoCast scheme [112], the authors propose the following retransmission probability calculation:

$p=\frac{2}{n_{b} \cdot 0.4}$

Table 2

Fixed probabilistic schemes.

\begin{tabular}{|c|c|c|}
\hline Probabilistic scheme & Forwarding probability & Application \\
\hline GOSSIP1(p) $[41,42]$ & $p=p_{i}$ & MANET \\
\hline $\operatorname{GOSSIP} 1(\mathrm{p}, \mathrm{k})[41,42]$ & $\begin{array}{lll}p=1 & \text { if } & n h<k \\
\text { else } & p=p_{i}\end{array}$ & MANET \\
\hline GOSSIP_DIR1 [104] & $\vec{p}=\left(p_{1}, \ldots, p_{N}\right)$ & MANET \\
\hline
\end{tabular}




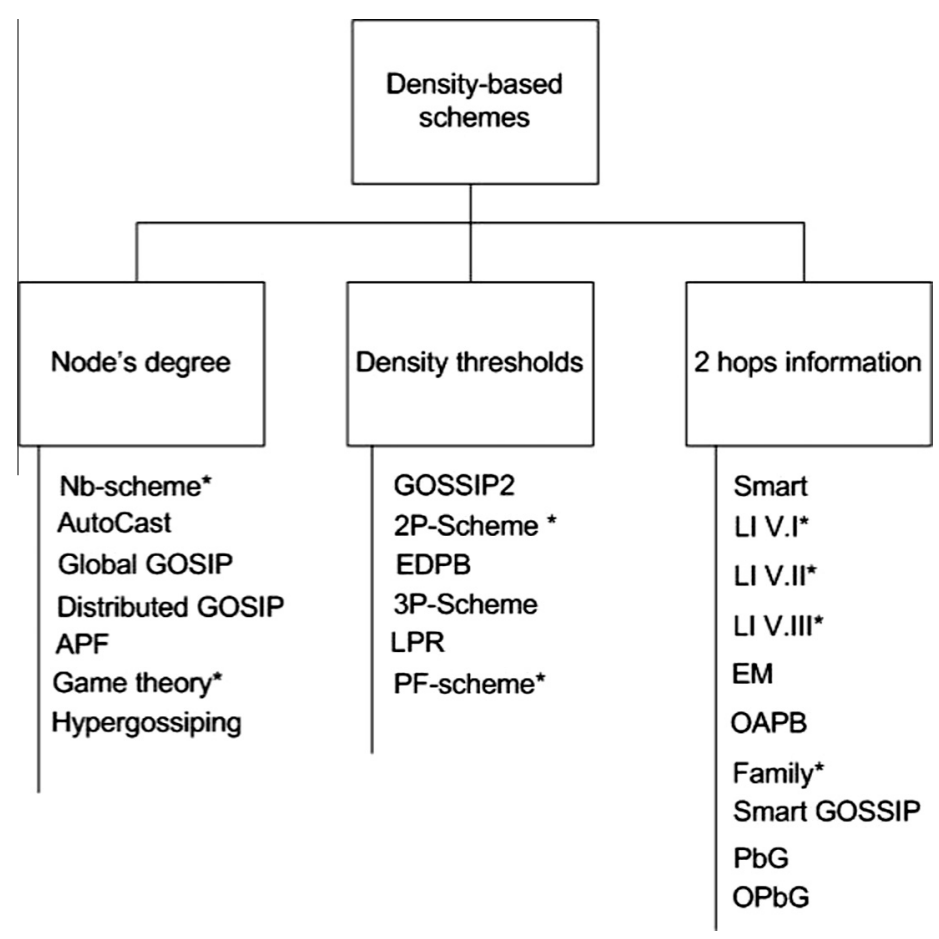

Fig. 3. Classification of density-based schemes.

In addition, nodes broadcast messages periodically in order to improve the reliability of the proposed scheme. The broadcast interval is also adjusted based on the number of neighbors:

$$
T=\frac{n_{b}}{\alpha}
$$

where $\alpha$ is a constant, which defines the number of broadcasts per second.

In Global GOSSIP [70], the authors demonstrated that if the nodes forward incoming packets with a probability $p=\frac{\theta^{-1}(\gamma)}{\phi}$ in a network with average degree $\phi>\theta^{-1}(\gamma)$, the probability of a successful broadcast procedure is $\gamma$. Where $\gamma$ is the target gossip coverage level (target reachability), $\phi$ the average node degree, and $\theta^{-1}(\gamma)$ is the minimum average degree so that the fraction of nodes in the largest connected component of a network with infinite size is at least $\gamma$. The average node's degree $\phi$ is defined as $\phi=\lambda \pi r, \lambda$ is the intensity of homogeneous Poisson process $H_{\lambda}$, which is used to distribute nodes in the network. Although this scheme is suitable from the theoretical point of view, it makes assumptions on the level of knowledge available at individual nodes, which would either incur huge cost or impossible to attain. First, nodes have to know the average node degree of the network $\phi$, which is a global information and it can be costly in terms of information exchanges. Another issue is how nodes can know the value of $\theta^{-1}(\gamma)$ in a real network.

An improved version of Global GOSSIP is presented as Distributed GOSSIP in [70]. Nodes use the node's number of neighbors $n_{b}$ instead of using the average node degree $\phi$ in the network. According to Distributed GOSSIP, nodes have to forward the incoming packets with a probability $p=\min \left(1, \frac{\theta^{-1}(\gamma)}{n_{b}}\right)$ in order to achieve the target coverage level $\gamma$. In addition, the authors estimated that above a certain average degree $\phi_{u}$, distributed GOSSIP outperforms global GOSSIP since the coverage level achieved will be higher than the target coverage level. However, this scheme still has the problem of the nodes having to obtain the value for $\theta^{-1}(\gamma)$.

In $[44,45]$ (APF), the forwarding probability is also adjusted using the node's number of neighbors. The main difference from the previous schemes is that two probability boundaries $p_{\max }$ and $p_{\min }$, are defined by the present scheme. Consequently, the forwarding probability is within the interval $\left(p_{\min }, p_{\max }\right)$. The forwarding probability is calculated by the following expression:

$p=\max \left(p_{\max }\left(\frac{p_{\max }^{n_{b}-1}-p_{\max }^{n_{b}}}{1-p_{\max }}\right), p_{\min }\right)$

The authors conducted simulations to determine the optimal values of $p_{\max }$ and $p_{\min }$.

In [80] the authors apply game theory [86], and proposed a framework called Forwarding Dilemma Game (FDG). The game is played whenever a node receives a packet from other nodes in the network. The player (a node) has two strategies: (1) forward the packet or (2) drop the packet. The FDG has three components: (1) the number of players, which is the number of nodes receiving 
the broadcast packet, (2) the forwarding cost C, and (3) the network gain factor G. The FDG is formally defined as follows:

$G=\left\{n_{b},\left(S_{i}\right)_{i \in n_{b}},\left(U_{i}\right)_{i \in n_{b}}\right\}$

where $S_{i}$ is the strategy set, and $U_{i}$ is the utility function. The strategy set of a node $i$ is the number of actions that the node can carry out. When $S_{i}=1$ the node forwards the incoming packet, by contrast, if $S_{i}=0$ the node drops the packet. Moreover, each node will receive utility function $U_{i}$ on choosing a strategy set value $S_{i}$. The authors proposed the mixed Nash equilibrium [86] to derive the forwarding probability.

$p=1-\left(\frac{C}{G}\right)^{\frac{1}{n_{b-1}}}$

According to (5), the probability decreases as the number of neighbors $n_{b}$ increases, in the limit $n_{b} \rightarrow \infty, p \rightarrow 0$. The authors assumed $C=1$ and demonstrated that the value of $\mathrm{G}$ should satisfy $3 \leqslant \log (G) \leq 6$.

In [57] (Hypergossiping), the authors proposed an adaptive broadcasting scheme intended for partitioned MANETs. Whenever a partition occurs the broadcasting procedure stops because of the impossibility of new retransmissions. Identifying a partition is challenging due to the lack of global point of view of nodes in a wireless network. The authors proposed implementing a list of "Last Broadcast Received" LBR at nodes to identify partitions. The LBR lists include the last few broadcast packets received by a given node. The main assumption is that two nodes within the same partition will have similar LBR lists, and if two nodes have dissimilar LBRs, they will be located in different partitions. The LBRs are exchanged by nodes using hello packets, so nodes can discern if they are joining a new partition by comparing the received LBR with its own LBR. In order to reduce the overhead of hello packets, the LBR is only necessary if a new neighbor is detected. The performance of Hypergossiping can be divided into two strategies, (1) intra-partition forwarding and (2) broadcast repetition. The intrapartition forwarding consists of disseminating the broadcasting messages efficiently within the node's partition. The forwarding probability is adjusted using the node's density. They developed a calibration procedure to get and store in a look up table the optimum values for forwarding probability according to the node's neighbors. In addition, nodes do not retransmit immediately after receiving an incoming packet, they delay the retransmissions for a random time.

\section{(b) Density thresholds:}

In [42] GOSSIP2, a scheme based on a density threshold is proposed. This density threshold is based on the number of neighbors $n_{b}$. Two values of forwarding probabilities $p_{h}$ and $p_{l}$ are used. When a given node has $n_{b}$ higher than $n_{c}$ (density threshold), the probability is $p_{l}$, otherwise, the probability is $p_{h}$, where $p_{h}>p_{l}$. As a consequence, nodes with low degree have more probability to forward the incoming packets. The main shortcoming of this scheme is how the nodes could determine the optimum values of $n_{c}, p_{h}$ and $p_{l}$. These values can vary drastically depending on the application scenario.

A similar idea is used in 2P-Scheme [13,119], but in this scheme the average number of neighbors $n_{h}$ of a given node is used as the density threshold $\left(n_{h}=n_{c}\right)$.

In [3] (EDPB), the average number of neighbors in the network $n_{a v g}$ is used as a threshold instead of $n_{h}$. It is worth pointing out the difference between $n_{a v g}$ and $n_{h}$ at this point. While $n_{\text {avg }}$ is calculated using global information (6) so that every node in the network is considered, $n_{h}$ is calculated locally at a node without using any global information.

Other authors proposed to use more than one density threshold as in 3P-Scheme [3], in smart scheme [118], and in Level Probabilistic Routing (LPR) [127]. In 3Pscheme, the authors classified the nodes based on the number of neighbors. The average number of neighbors of nodes in a network can be calculated as follows using global information:

$n_{\text {avg }}=\frac{\sum_{i=1}^{N} n_{b_{i}}}{N}$

Moreover, the expected maximum and minimum average values for the number of neighbors can be calculated as follows:

$n_{a v g_{\min }}=\frac{\sum_{i=1}^{M_{\text {min }}} n_{b_{i}}}{M_{\min }}$ where $n_{b_{i}} \leqslant n_{\text {avg }}$
$n_{a v g_{\max }}=\frac{\sum_{i=1}^{M_{\max }} n_{b_{i}}}{M_{\max }}$ where $n_{b_{i}}>n_{\text {avg }}$

where $M_{\min }$ and $M_{\max }$ are the number of nodes with a number of neighbors below $n_{\text {avg }}$ and above $n_{\text {avg }}$ respectively. A node $i$ is considered to be in high density area if $n_{b_{i}}>n_{a_{\text {g }} \text { max }}$ so a low forwarding probability will be given to node $i$. In contrast, if $n_{b_{i}}<n_{a v g_{\text {min }}}$ the node will be considered to be in a sparsely populated area so a high forwarding probability will be assigned to it. In summary the forwarding probability is adjusted as follows:

$p=p_{1} \quad$ if $\quad n_{b} \leqslant n_{a_{\text {min }}}$

$p=p_{2} \quad$ if $\quad n_{a v g_{\min }}<n_{b} \leqslant n_{a v g_{\max }}$

$p=p_{3}$ if $n_{b} \geqslant n_{a v g}$

where $p_{1}>p_{2}>p_{3}$.

In smart scheme, three threshold values are defined to determine the forwarding probability [118] and up to four forwarding probability values are defined. As in the 3P-Scheme [3], the main drawback of this method is that it uses global density measures like $n_{a v g}, n_{a v g_{\min }}$, and $n_{a v g_{\max }}$.

In LPR scheme [127], nodes are divided into four groups based on their degrees. The group 1 is composed of nodes with a degree higher than all their neighbors. In contrast, nodes with a lesser degree than their neighbors are included in group 4 . The group 2 is composed of nodes with most of the neighbors having a lower degree than them. Finally, the rest of nodes are included in group 3. Nodes in each group have different forwarding probabilities, which are $p_{1}, p_{2}, p_{3}$ and $p_{4}$ respectively. The forwarding probabilities are selected such that $p_{1} \geqslant p_{2} \geqslant p_{3} \geqslant p_{4}$. 
In [51] (PF), the authors proposed a piecewise probability function with the number of neighbors as the independent variable, and the forwarding probability as the dependent variable. The number of neighbors $n_{b}$ is compared to $n_{a v g}$ according to the deployed topology, it can be estimated by using the following equation:

$n_{\text {avg }}=(N-1) \frac{\pi r^{2}}{A_{\text {net }}}$

where $A_{\text {net }}$ is the size of the network scenario. According to $n_{\text {avg }}$, a given node is in a dense area if $n_{b}>n_{\text {avg }}$, otherwise it is considered to be in a sparse area. A forwarding probability function is defined based on the value of $n_{b}$ as given below:

$p=1 \quad$ if $\quad n_{b}<n_{\text {avg }}$

$p=p_{i} \quad$ if $\quad n_{a v g}<n_{b} \leq 2 n_{a v g}$

$p=\frac{p_{i}}{2} \quad$ if $\quad 2 n_{\text {avg }}<n_{b} \leq 3 n_{\text {avg }}$

$p=\frac{p_{i}}{3} \quad$ if $\quad 3 n_{a v g}<n_{b} \leq 4 n_{a v g}$

$p=\frac{p_{i}}{r} \quad$ if $\quad \theta n_{\text {avg }}<n_{b} \leqslant(\theta+1) n_{\text {avg }}$

where $p_{i}=0.7$, this value is chosen for ensuring certain reachability. The forwarding probability decreases as the number of neighbors of a given node increases. Although the results obtained from performance are good, the approach is very dependent on the deployed scenario. Moreover, the value of $p_{i}=0.7$ may also be dependent on the deployed scenario.

\section{(c) 2 hops information:}

In [99], the authors proposed three probabilistic approaches based on the number of neighbors at one-hop and two-hops levels of a node (LI.I, LI.II, and LI.III). In version I (LI.I), the forwarding probability is adjusted according to the number of two-hops neighbors that can only be reached through one-hop neighbors.

$p=\frac{1}{n_{b}} \sum_{k=1}^{n_{b}} n\left(x_{i}, x_{k}\right)$

where $n\left(x_{i}, x_{k}\right)$ is the set of two-hops neighbors that can only be reached through one-hop neighbor $x_{k}$ from a given node $x_{i}$ (for $k=1,2,3, \ldots, n_{b}$ and $\left.i=1,2,3, \ldots, N\right)$. The version I (LI.I) tries to avoid isolated nodes by giving a high probability value to those nodes that are the only possible way to connect to two-hops neighbors. In version II, the retransmission probability is calculated as the fraction of one-hop neighbors within two-hops coverage.

$p=\frac{n_{b}}{n_{b}+n_{b}^{2 h}}$

where $n_{b}^{2 h}$ is the set of two-hops neighbors of a given node $x_{i}$. In version II (LI.II), higher the number of one-hop neighbors, higher the forwarding probability. In the third version (LI.III), the forwarding probability is calculated as the fraction of two-hops neighbors within two-hops coverage. $p=\frac{n_{b}^{2 h}}{n_{b}+n_{b}^{2 h}}$

In version III, higher the number of two-hops neighbors, higher the forwarding probability.

In [97], the authors proposed a new expansion metric to measure how the density of the network changes over the propagation path of the broadcast process. The expansion metric is calculated as follows:

$E M=\frac{n_{b}^{2 h}}{n_{b}}$

This metric is used by nodes hop by hop along the broadcast path to recalculate the forwarding probability as follows:

$$
\begin{array}{lll}
p=p_{i}+p_{E M} & \text { if } & E M_{t}<E M_{t-1} \\
p=p_{i}-p_{E M} & \text { if } & E M_{t}>E M_{t-1} \\
p=p_{i} & \text { if } & E M_{t}=E M_{t-1}
\end{array}
$$

where $p_{i}$ is a constant probability value used to ensure high reachability and $p_{E M}$ is a probability value that is added or subtracted depending on the EM values, $E M_{t}$ is the expansion metric of the current node and $E M_{t-1}$ is the expansion metric of the previous node. If $E M_{t}<E M_{t-1}$, it means that the network density is decreasing along the broadcast path so the term $p_{E M}$ is added to increase the forwarding probability. On the other hand, if $E M_{t}>E M_{t-1}$, the network density is increasing along broadcast path so the forwarding probability should be reduced.

In OAPB [8], the authors combined the three versions proposed in [99] (LI.I, LI.II, and LI.III). These versions are averaged to achieve a forwarding probability value based on up to two-hops neighbor information. The retransmission probability is calculated as follows:

$p=\frac{p_{1}+p_{2}+p_{3}}{3}$

where $p_{1}, p_{2}$, and $p_{3}$ are calculated using (11)-(13) respectively. In addition, the authors proposed delaying the retransmissions based on the $p$ value obtained at each node by,

$\Delta(t)=\Delta(t)_{\max }(1-p)+\delta$

where $\Delta(t)_{\max }$ is the maximum delay and $\delta$ is a random variable whose value is in the order of milliseconds.

In [53] (Family classification), the node's neighbors are grouped into three levels: parent nodes (upper level), sibling nodes (same level), and child nodes (lower level). This classification is made during an initial phase in which the nodes use simple flooding to collect neighbor information. The source node's 1 hop neighbors are considered sibling nodes, whereas the source node's 2 hops neighbors are considered child nodes. Intuitively, the more siblings a node has, the less necessity for retransmission as all of the node's children may have received a broadcast packet. Although the node may not forward the packet, its children will likely to have received the packet from aunt nodes (siblings of the parent). This scheme is composed of three phases, (1) nodes collect neighboring information by using hello packets, (2) nodes determine their level within the topology tree and compute their relationship with all their 
neighbors, and (3) nodes determine their forwarding probabilities based on the number of child and sibling nodes. In summary, the forwarding probability is proportional to the number of child nodes and inversely proportional to the number of sibling nodes as shown in (18).

$$
\begin{array}{lll}
p=0 & \text { if } & n_{\text {child }}=0 \\
p=\max \left[\left(\frac{1}{n_{\text {sibling }}+1}+p_{i} \frac{n_{\text {child }}}{N}\right), 1\right] & \text { if } & \text { otherwise } \\
p=1 & \text { if } & n_{\text {child }}>0, n_{\text {sibling }}=0
\end{array}
$$

where $n_{\text {child }}$ is the number of child nodes, $n_{\text {sibling }}$ is the number of sibling nodes, and $p_{i}$ is an initial probability depending on the node density. The authors used $p_{i}$ within the range $[1,0.5]$.

A similar scheme is presented in Smart Gossip [60,61], which is also based on the dependencies among nodes in a wireless network. The main difference between Smart Gossip and the previous scheme (Familiy classification) is that in Smart Gossip nodes adjust the forwarding probability in order to achieve a target reliability level. One important feature of this scheme is that it works on a per originator basis so a node $X$ chooses a probability $p$, which is independent of the incoming packet. Notice that, most broadcasting schemes work on a packet basis since nodes recompute the forwarding probability each time a new packet is received. Furthermore, this scheme does not rely on hello packets. It is based on proximity overhearing of broadcast messages. As a consequence, nodes collect the neighboring information as they receive the broadcast packets. Overhearing these broadcast messages, a node can deduce what type of node the sender is. Therefore a node can distinguish between parent nodes, sibling nodes, and child nodes. The broadcast packets contain a parent identifier field $p_{i d}$ and a required gossip probability field $p_{\text {required }}$. The required gossip probability that a node is related to the target reliability that a node advertizes to its parents. The gossip probability $p$ is calculated by the children of a node. The $p$ of a node is given by $p=\max \left(p_{\text {required }}^{i}\right)$. So the $p$ of node $X$ is determined by the maximum $p_{\text {required }}$ announced by any child $i$ of $X$. This scheme starts with an initial phase in which the nodes do not know their dependencies so they forward with a probability $p=1$. Over time, nodes recompute their $p_{\text {required }}$ so that $p$ can be recomputed. As mentioned, each node advertizes a $p_{\text {required }}$. This probability value is adjusted according to a defined reliability level. The authors presented the average reception percentage $\tau_{\text {arp }}$ as a metric to assign a target reliability level. The reception percentage of a node $X$, with respect to an originator node $O$, is the percentage of messages originated at $O$ and received at $X$. The average reception percentage $\tau_{\text {arp }}$ is the reception percentage, averaged over all nodes in the network. For a target $\tau_{\text {arp }}$ the authors translate it into a per-hop reception probability $\tau_{\text {rel }}$. The main objective is that the node's children receive $\tau_{\text {rel }}$ fraction of the originator node's packets. Since the decision of a node to forward a packet is made independently, $\tau_{\text {rel }}$ can be estimated by solving the equation $\left(\tau_{\text {rel }}\right)^{\delta}=\tau_{\text {arp }}$, where $\delta$ is the network's diameter. If a node has only one parent, $p_{\text {required }}$ will be equal to $\tau_{\text {rel }}$. However, when a node has $k$ parents, then it suffices to assign a $p$, which ensures that the probability of at least one parent retransmits is greater than $\tau_{\text {rel }}$. As a result, the $p_{\text {required }}$ announced by a node with $k$ parents can be estimated as follows:

$$
\left(1-p_{\text {required }}\right)^{k}<\left(1-\left(\tau_{\text {rel }}\right)\right)
$$

Ensuring that condition (19) is met, the target reliability will be met for the determined service as well. In $[60,61]$ the authors also combined the proposed probabilistic scheme with a deterministic enhancement for when the packet loss is likely to happen. In this case, nodes insert a sequence number in the broadcast messages to check whether they miss any packet. If a given node misses any broadcast packet, it will explicitly request one of its parents to retransmit the missing packet.

This scheme presents several shortcomings that prevents its application to mobile networks [11]: (1) The reception percentage between two nodes is based on the quality of the link, however, in mobile networks mobility is the main reason for broken links. (2) The hierarchy is established in a static way and no renewal of neighborhood information is considered. Moreover, it is demonstrated in [11] that smart gossip fails when more than one source node is considered.

In $\mathrm{PbG}$ [11], the authors applied the aforementioned dependencies among nodes [60] to VANETs. They considered positioning data to calculate the relationships among nodes. These relationships take the direction of the dissemination process into consideration. As the proposed scheme is intended for vehicular networks, two possible directions can be considered. Fig. 4 illustrates the dependencies for a vehicular network composed of 4 nodes. In this example the hierarchy is built against driving direction. Vehicles are approaching the traffic congestion and they have to be informed. The node D is considered as the source node. An important difference of PbG from smart gossip is that in PbG nodes use Hello packets to collect positioning information. Since this scheme is aimed at VANETs, the dependencies among nodes must be updated frequently.

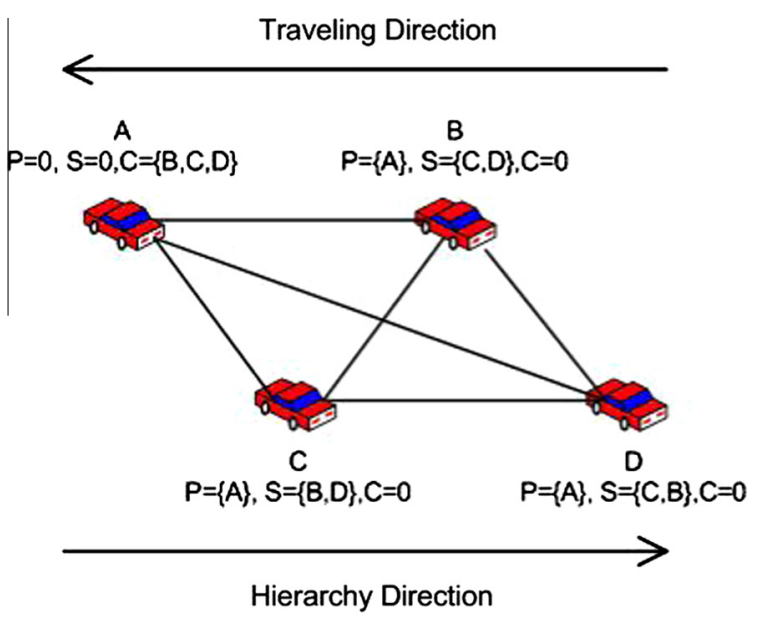

Fig. 4. Node's relationships in PbG. 
In [12] the PbG scheme is enhanced by including three extensions (OPbG). (1) Two table extension, this improvement solves the problem of two source nodes of smart gossip [60]. Nodes consider a different neighboring table for each possible directions. As a result, distinct dependencies are defined for each direction. (2) Network density extension, this reduces the forwarding probability for dense networks. The authors introduced an additional reduction factor red that depends on the network density. They conducted simulations to derive the following expression for red:

$\operatorname{red}\left(n_{b}\right)=\frac{0.5}{1+e^{-a\left(n_{b}-b\right)}}$

The parameters $a$ and $b$ are obtained by simulation. Finally, the reduction factor red is applied to the average reception percentage $\tau_{\text {arp }}$.

$\tau_{\text {arp }} i=\tau_{\text {arp }}-$ red

(3) Fallback mechanism extension, this is a counter-based [109] improvement to avoid collisions.

A summary of the reviewed density-based schemes is presented in Tables 3 and 4 .
3.2.1.2. Distance-based schemes. The schemes based on distance can be further divided into two main categories: area-based and location based schemes. In the former category, the main idea is to use the relative distance between two nodes to adjust the forwarding probability, so nodes do not need global information of other nodes in the network. These schemes can also be categorized as densitybased, RSS (Received Signal Strength), Euclidean distance, hop count, and hints-based (see Fig. 4). In density-based schemes, the relative distance between two nodes is estimated using the distribution of neighbors within a node's transmission range $[33,32,103]$. The main advantage of these schemes is that nodes do not need a positioning system. However, the validity of such estimation may depend on a constant distribution of nodes in the networks, an assumption that is always not true. In RSS-based schemes, nodes calculate the relative distance as a function of the received signal power $[69,114,115]$ (the readers are referred to [90] for further information on estimation techniques). In Euclidean distance-based schemes, nodes have to be equipped with a positioning system like a GPS in order to obtain the Euclidean distance between two nodes $[115,69]$. Alternatively, as the number of hops is the common metric used by routing protocols to measure the distance between the source node and the destination node [72], this can also be used by the probabilistic broadcast

Table 3

Adaptive non counter-based density-based probabilistic schemes I.

\begin{tabular}{|c|c|c|c|c|c|}
\hline Scheme & Subtype & Forwarding probability & Collected data & Adjusted parameters & Application \\
\hline nb-Scheme [33] & Degree & $p=\frac{k}{n_{b}}$ & $n_{b}$ & $k$ & MANET \\
\hline Autocast [112] & Degree & $p=\frac{2}{n_{b} \cdot 0.4}$ & $n_{b}$ & $\alpha$ & VANET \\
\hline Global GOSSIP [70] & Degree & $p=\frac{\theta^{-1}(\gamma)}{\phi}$ & $\phi$ and $\theta^{-1}(\gamma)$ & $\gamma$ & MANET \\
\hline Distributed GOSSIP [70] & Degree & $p=\frac{\theta^{-1}(\gamma)}{n_{b}}$ & $n_{b}$ and $\theta^{-1}(\gamma)$ & $\gamma$ & MANET \\
\hline APF $[44,45]$ & Degree & $(3)$ & $n_{b}$ & $p_{\max }$ and $p_{\min }$ & MANET \\
\hline Game theory [80] & Degree & $p=1-\left(\frac{C}{G}\right)^{\frac{1}{n_{b}-1}}$ & $n_{b}$ & $C$ and $G$ & MANET \\
\hline Two thresholds (GOSSIP2) $[41,42]$ & Thresholds & $\begin{array}{ll}p=p_{h} & \text { if } \quad n_{b}<n_{c} \\
\text { else } & p=p_{l}\end{array}$ & $n_{b}$ & $n_{c}, p_{h}$, and $p_{l}$ & MANET \\
\hline 2P-Scheme [3] & Thresholds & $\begin{array}{lll}p=p_{h} & \text { if } & n_{b}<n_{h} \\
\text { otherwise } & p=p_{l}\end{array}$ & $n_{b}$ & $p_{h}$ and $p_{l}$ & MANET \\
\hline EDPB [13,35] & Thresholds & $\begin{array}{lll}p=p_{h} & \text { if } \quad n_{b}<n_{\text {avg }} \\
\text { otherwise } & p=p_{l}\end{array}$ & $n_{b}$ & $p_{h}$ and $p_{l}$ & MANET \\
\hline 3P-Scheme [3] & Thresholds & $(8)$ & $n_{b}$ & $p_{1}, p_{2}, p_{3}$ & MANET \\
\hline $\mathrm{PF}[51]$ & Thresholds & $\begin{array}{ll}p=1 & \text { if } \\
\text { else } & p=\frac{p_{i}}{\theta}\end{array} \quad n_{b}<n_{\text {avg }}$ & $n_{b}$ & $p_{i}$ and $n_{a v g}$ & MANET \\
\hline LI.I [99] & 2 Hops & $p=\frac{1}{n_{b}} \sum_{k=1}^{n_{b}} n\left(x_{i}, x_{k}\right)$ & $n_{b}$ and $n\left(x_{i}, x_{k}\right)$ & - & MANET \\
\hline LI.II [99] & 2 Hops & $p=\frac{n_{b}}{n_{b}+n_{b}^{2 h}}$ & $n_{b}$ and $n_{b}^{2 h}$ & - & MANET \\
\hline LI.III [99] & 2 Hops & $p=\frac{n_{b}^{2 h}}{n_{b}+n_{b}^{2 h}}$ & $n_{b}$ and $n_{b}^{2 h}$ & - & MANET \\
\hline
\end{tabular}

Table 4

Adaptive non counter-based density-based probabilistic schemes II.

\begin{tabular}{|c|c|c|c|c|c|}
\hline Scheme & Subtype & Forwarding probability & Collected data & Adjusted parameters & Application \\
\hline EM [97] & 2 Hops & $(15)$ & $n_{b}$ and $n_{b}^{2 h}$ & $p_{i}$ & MANET \\
\hline OAPB $[8]$ & 2 Hops & $p=\frac{p_{1}+p_{2}+p_{3}}{3}$ & $n_{b}$ and $n_{b}^{2 h}$ & - & VANET \\
\hline Family classification [53] & 2 Hops & $(18)$ & $n_{b}, n_{\text {child }}, n_{\text {sibling }}$, and $N$ & $p_{i}$ & MANET \\
\hline Smart GOSSIP [60] & 2 Hops & $\left(1-p_{\text {required }}\right)^{k}<\left(1-\left(\tau_{\text {rel }}\right)\right)$ & $\tau_{\text {rel }}$, ParentSet, SiblingSet, ChildSet & $\tau_{\text {arp }}$ & WSN \\
\hline OPbG [12] & 2 Hops & $\left(1-p_{\text {required }}\right)^{k}<\left(1-\left(\tau_{\text {rel }}\right)\right)$ & $\tau_{\text {rel }}$, ParentSet, SiblingSet, ChildSet & $\tau_{\text {arp }}$ red & VANET \\
\hline
\end{tabular}


schemes to determine the distance between two nodes. In hints-based schemes, nodes estimate the relative distance between two nodes based on the history of connectivity of the two nodes [17].

On the other hand, in location-based schemes, nodes have to implement a location service so that nodes can exchange positioning data in order to create a map of the network.

3.2.1.2.1. Area-based schemes. This subsection reviews the main area-based schemes found in the literature that fall into the classification made in Fig. 5.

\section{(a) Density-based:}

There are several area-based schemes that estimate the relative distance between two nodes using density information such as BNR [33], B-overlap [103], PPR [103], and Jaccard distance [98].

In BNR scheme [33], the authors studied the intersection of two nodes' coverage areas, three zones $Z_{a}, Z_{b}$, and $Z_{c}$ can be defined as shown in Fig. 6 . The zone $Z_{a}$ is the communication area covered only by node $i$, The zone $Z_{b}$ is the communication area covered only by node $j$, and the zone $Z_{c}$ is the communication area covered by both nodes $i$ and $j$. However, these areas can be characterized by the number of mobile nodes inside them. The authors defined the ratio $\mu=\frac{N_{b}}{N_{a}+N_{c}}$, where $N_{a}, N_{b}$, and $N_{c}$ are the number of mobile nodes inside the areas $Z_{a}, Z_{b}$, and $Z_{c}$ respectively. If the node $i$ is considered as the source node

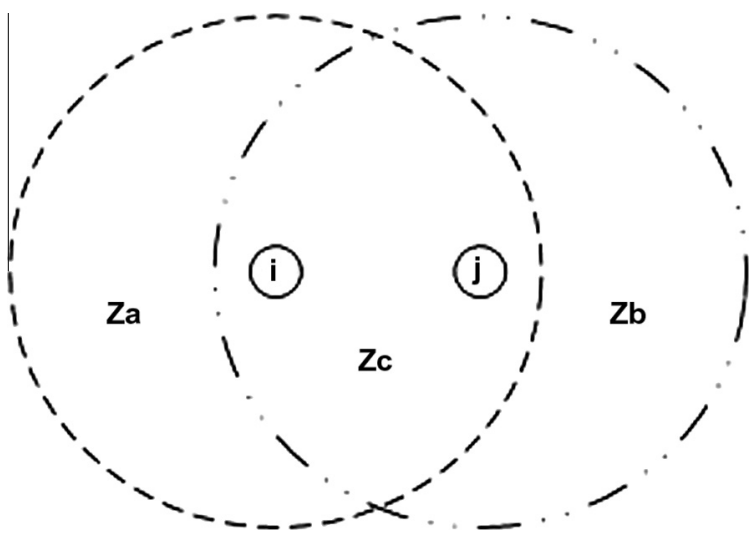

Fig. 6. Intersection of the radio transmission areas of two nodes.

and the node $j$ as the destination node, $\mu$ gives the ratio of unexplored nodes divided by the redundant nodes. This ratio becomes larger as the Euclidean distance between the two nodes becomes larger (if a uniform distribution of nodes is considered). In BNR, the forwarding probability is calculated by the following expression:

$p=\frac{A-\alpha}{M^{\sigma}} \mu^{\sigma}+\alpha$

where $A$ and $\alpha$ are the roof and floor probability levels. The authors defined $A=1$ and $\alpha=0, \sigma$ as the coefficient of convexity, and $M$ as a constant, which represents the

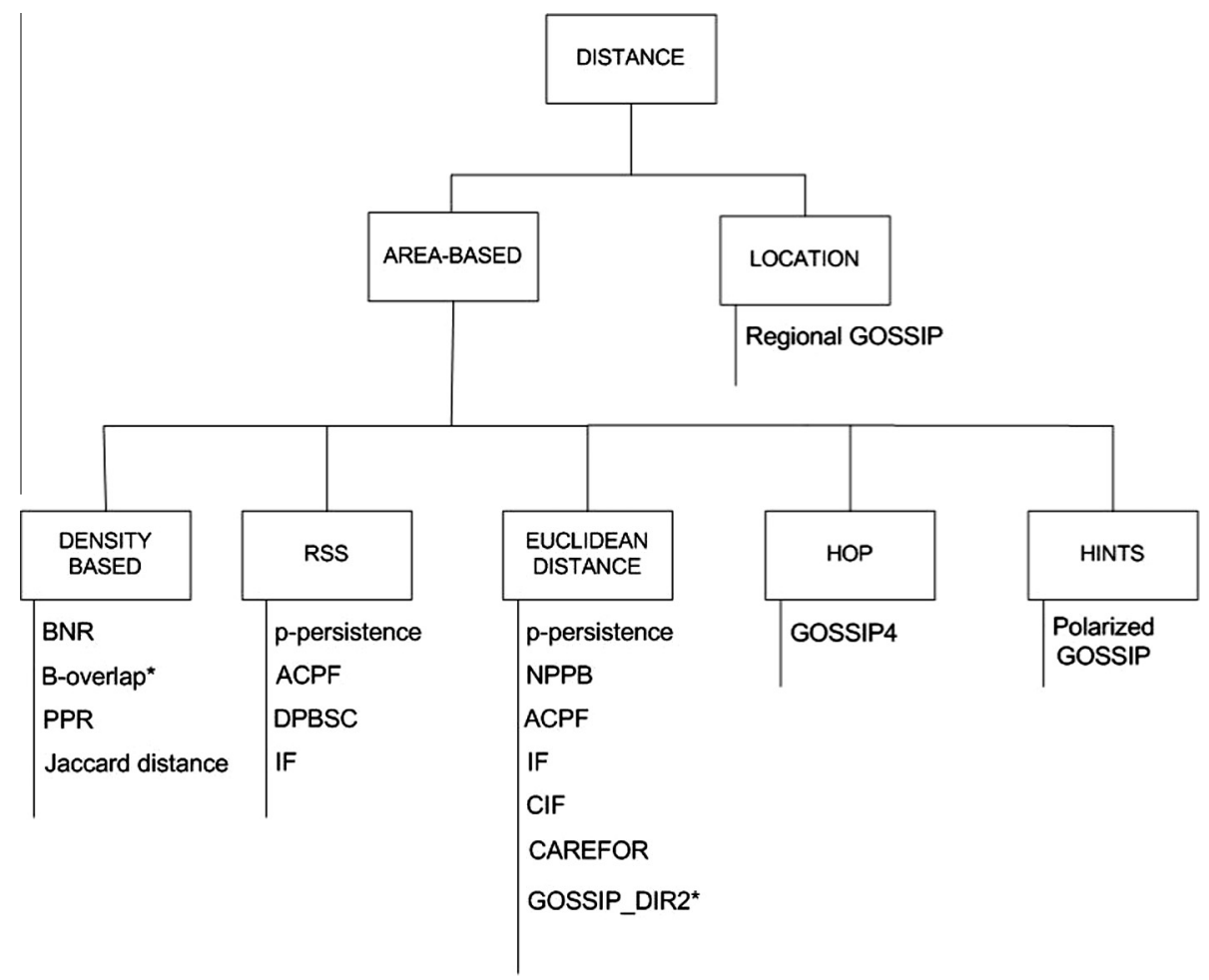

Fig. 5. Classification of distance-based schemes. 
maximum value of $\mu, M \cong 0.601$. Three values of $\sigma$ are considered $\sigma=1,2$, and 3 resulting in three different probability functions.

In B-overlap scheme [103], the authors compute the proportional ratio (PR) of the overlap between the source and the destination nodes to the total broadcast area of the source node as:

$S=\int_{\frac{r}{2}}^{r} \sqrt{r^{2}-x^{2}} d x$

Identical transmission ranges for the two nodes is assumed in (23), and $S_{\max }=0.41 \pi r^{2}$. This limit is achieved when the two nodes are located at the border of the transmission range. The authors defined the number of nodes in the overlap area (NNOA) which can be calculated as the node density multiplied by the proportional relationship between the broadcast area and the overlap area. The NNOA can be expressed as:

$$
\mathrm{NNOA}=\mathrm{LND} \cdot 0.41
$$

where LND is the local node density which can be calculated by exchanging hello packets. The source node can calculate the forwarding probability, using NNOA, as:

$p=\frac{\mathrm{DR}}{\mathrm{NNOA}}$

where DR is the desired number of retransmissions per overlap area. As a result, if each node in the overlap area retransmits with a probability $p$, the desired number of retransmissions is generated. In this scheme, nodes can adjust the reliability by tuning the parameter DR. If higher reliability is desired, the DR value should be higher. It is worth pointing out that this scheme also assumes that the nodes are deployed uniformly. In addition, in [103] the authors propose PPR, a scheme, which favors nodes located at the perimeter of node's transmission range. Similar to the previous scheme, this is also based on the local density to estimate the number of nodes at the perimeter zone of a node's transmission area. The source node can ignore nearby nodes by computing the desired retransmission volume based on the occupancy of a rim of some width $\varepsilon$ around the perimeter, see Fig. 7 .

In this scheme, nodes calculate the forwarding probability as

$p=\frac{\mathrm{DR}}{\left(1-\varepsilon^{2}\right)} \mathrm{LND}$

where LND is the local node density, and a rim of width $r(1-\varepsilon)$ is considered, $D R$ is the desired retransmissions along the rim.

In [98], the authors use the Jaccard distance [46] to estimate the Euclidean distance between two nodes. The main idea is to favor dissimilar nodes in order to reduce redundant messages. The Jaccard distance is calculated using one hop neighboring information, considering two neighbors i and $\mathrm{j}$ (see Fig. 8) the Jaccard distance is expressed as:

$J d=1-\frac{a_{1}}{a_{1}+a_{2}+a_{3}}$

where $a_{1}$ represents the common neighbors of the nodes $i$ and $j, a_{2}$ is the number of neighbors of the node $i$ that are

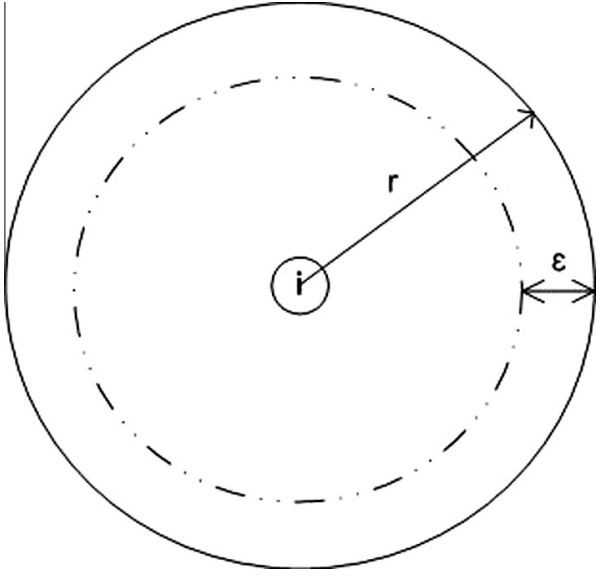

Fig. 7. Proportional perimeter scheme.

not neighbors of the node $j$, and $a_{3}$ is the number of neighbors of the node $j$ which are not neighbors of the node $i$. In order to calculate the Jaccard distance, nodes include their lists of neighbors in the hello packets.

The authors demonstrate in [98] that the Jaccard distance is correlated to the Euclidean distance. They assigned a linear probability based on the Jaccard distance between two nodes.

$p=J d$

where $J d$ is calculated using (26) and $J d \in[0,1]$. In addition, they also adjusted the forwarding delay based on the Jaccard distance, so nodes located further away from a node have a lower retransmission delay.

\section{(b) Euclidean distance-based and RSS-based:}

In this subsection we have combined Euclidean distance-based and RSS-based schemes since in many aspects the schemes are similar and the only difference is the use of RSS levels instead of the Euclidean distance.

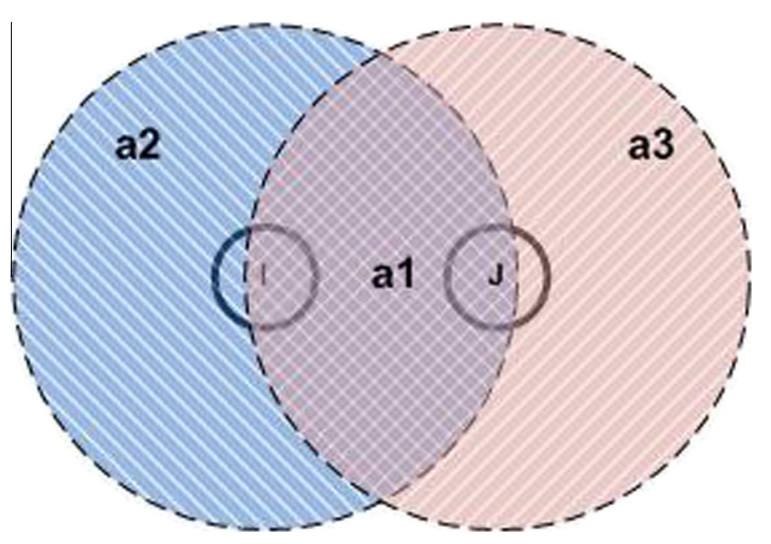

Fig. 8. Jaccard distance in MANETs. 
In $[115,114]$ (weighted $p$-persistence) the basic forwarding scheme based on the Euclidean distance is presented as:

$p=\frac{d_{i j}}{r}$

The proposed p-persistence is enhanced in $[114,115]$ by proposing the slotted $p$-persistence. In this scheme the retransmissions are delayed according to the relative distance between the sender and the receiver. The assigned time slot $T_{S i j}$ is calculated as follows:

$T_{s i j}=S l o t_{i j} \times l_{d}$

where $l_{d}$ is the estimated link propagation delay and $S{ } t_{i j}$ is the assigned slot number expressed as,

Slot $_{i j}=N_{\text {slot }}-\left\lceil\frac{d_{i j} \times N_{\text {slot }}}{r}\right\rceil$

where $N_{\text {slot }}$ is the pre-determined number of slots. The authors also proposed using the RSS level to estimate the distance between nodes.

An extension of the p-persistence scheme is presented in [130] (NPPB), the authors define that a node have a forwarding probability higher than 0.5 , when the weighted p-persistence is used up to $\frac{3}{4}$ of a node's neighbors. As a result, in a dense network, the effect of weighted persistence in saturated networks is not perceptible. They proposed an $n$ th-powered probabilistic scheme to further reduce the forwarding probability in dense VANETs. In NPPB the forwarding probability is calculated as,

$p=\left(\frac{d}{r}\right)^{k}$

where $k$ is the exponent for controlling the forwarding probability in NPPB. The larger the value of $k$, higher the concentration of retransmission nodes to the border of the node's transmission range. A similar approach of NPPB is proposed in [27] namely polynomial broadcast.

On the other hand, there are several schemes that consider the overlapping transmission areas between two neighbor nodes. In [69] (ACPF), the authors evaluated the overlapped transmission areas of two neighbor nodes $i$ and $j$ (see Fig. 9). This shared area $S(i, j)$ is represented in Fig. 9.

The shared area $S(i, j)$ can be calculated as,

$S(i, j)=\pi r^{2}+d \cdot \sqrt{r^{2}-\frac{d^{2}}{4}}-\frac{\arccos \frac{d}{2 r}}{90} \pi r^{2}$

The maximum value of $S(i, j)$ is $S_{\max }=0.61 \pi r^{2}$ and it is achieved when $d=r$. The authors also proposed estimating $d(i, j)$ using the received power. In ACPF the forwarding probability is calculated based on $S(i, j)$ as follows:

$p=p_{i} e^{k^{\frac{S(i, j)-S \max }{\pi r^{2}}}}$

The parameter $k$ is used to control the forwarding probability. A similar approach is proposed in [111] (DPBSC), where the authors used the additional coverage of rebroadcast to determine the forwarding probability. When a node receives a broadcasting packet, it refers to its additional coverage of rebroadcast to determine the rebroadcast probability. If the packet is received for the first time, the node applying DPBSC uses its coverage area to determine its rebroadcast probability as follows:

$$
\begin{array}{ll}
p=\frac{S(i, j)}{S_{\max }(i, j)} & \text { if } \quad 0<d<r \\
p=0 & \text { otherwise }
\end{array}
$$

In $[89,87,88]$ (Irresponsible Forwarding, IF), a scheme that combines the distance between two nodes and the density of nodes to calculate the forwarding probability is presented for VANETs. The forwarding probability is calculated using the relative distance between two vehicles and the spacing distribution of the vehicles in the network. For every possible spacing distribution, the forwarding probability is calculated using the following expression:

$p=\left(1-F_{x}(z-d)\right)^{1 / k}$

where $k$ is a shaping parameter and $F_{x}(z-d)$ is the cumulative distributed function (CDF) of a random variable $X$ representing the space between two vehicles. In [89], the authors used an exponential spacing distribution so the forwarding probability is:

$p=e^{-\frac{\varphi_{s}\left(r-d_{i j}\right)}{k}}$

where $\varphi_{s}$ is the spatial density distribution of nodes and in [28] IF is evaluated on 802.11 MAC protocol. In [87] IF is analyzed under general inter-vehicle spacing distributions. Recently in [88], the authors evaluated the IF scheme under real inter-vehicle spacing distributions. They used real traffic traces obtained from Berkeley Highway Laboratory. The main shortcoming of this scheme is how the nodes can be aware of the spacing distribution using only local information without a central system. The authors proposed using the historical traffic data to estimate the spacing distribution. The main assumption is that the spacing distribution is repeated over time. Furthermore, the authors extended the IF scheme applying the silencing technique in [27]. In IF with silencing whenever a node transmits a broadcast packet, it silences the rest of nodes in the same transmission area.

Despite the good results of IF [28], the authors pointed out a shortcoming of IF scheme in high density scenarios. The problem occurs when there are several nodes located at the same high distance from the source node, there will be several nodes with a high forwarding probability; and as a result of this situation, there will be a high probability of collision of messages. To avoid this problem, the authors extended the IF scheme by introducing the concept of Ephemeral Cluster (EC). They denoted this new scheme as Cluster-based Irresponsible Forwarding (CIF). In CIF, an EC is formed whenever there are several nodes located closely to each other in the sender's transmission range during a short period of time. Among the nodes forming the $\mathrm{EC}$, it is sufficient to disseminate the message if only one of them retransmits.

In [77] the CAREFOR protocol is proposed. Although CAREFOR is based on IF, the authors in [77] stated that the assumption that each node in the network has the same transmission range may not be real. Consequently, 


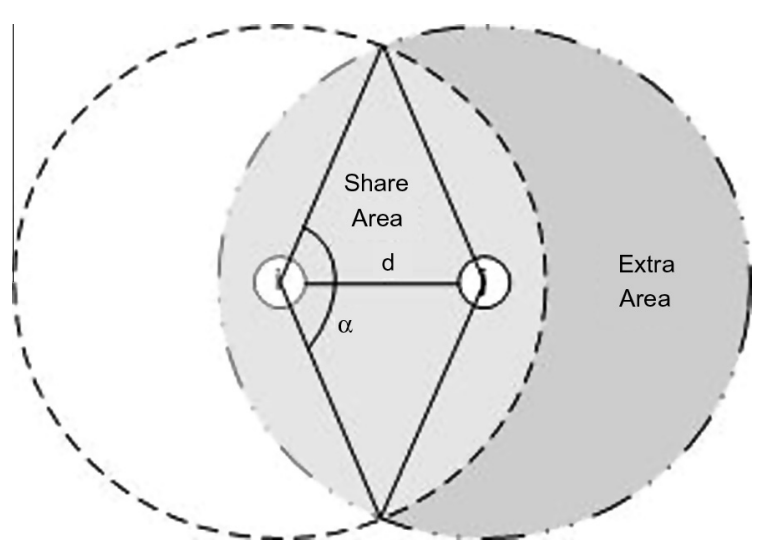

Fig. 9. Share and extra area of two nodes.

they modified the above Eq. (35) by including the transmission ranges of the nodes in the vicinity of the source node as follows:

$p=e^{-\frac{\varphi_{S}\left(r-d_{i, j}\right)}{k} r_{i}}$

being $r_{i}$ the radio transmission range of the $i$ th receiving node. It is noticeable that the ratio $\frac{r}{r_{i}}$ accounts for the differences in the nodes' transmission ranges. One of the main differences of CAREFOR from IF is that CAREFOR uses two hops information to calculate the forwarding probability. In addition, the authors also pointed out the problem of possible collisions for nodes located at higher distances from the sender as exhibited by IF scheme. This issue is aggravated if nodes with different transmission ranges are considered. In order to resolve this issue, the authors defined the following collision threshold based on density and the node's radio transmission range:

$T h_{\text {coll }}=1-e^{-\varphi_{s} r_{i}}$

The authors demonstrated that using the above collision threshold (37) the collision probability can be reduced. The mechanism of CAREFOR algorithm to avoid collisions is to compare the results of the collision threshold (37) with the collision probability at each node, which can be calculated as:

$P_{\text {coll }}=P_{\text {busy }}\left(1-P_{t}\right)$

Being Pbusy the probability of at least one node retransmits a given message and Pt the probability that there is only one node using the channel at this time. Both Pbusy and $\mathrm{Pt}$ are calculated as follows:

$P_{\text {busy }}=1-(1-p)^{(n-1)}$

$P_{t}=\frac{1-(1-p)^{(n-1)}}{P_{\text {busy }}}$

where $p$ is the forwarding probability calculated using (36) and $n$ is the number of nodes interfering with each other's transmission. According to [77], the performance of CAREFOR is divided into three phases: 91) RTB transmission phase, in this phase the source node sends a Request-to-Broadcast (RTB) control packet, including local information such as the GPS coordinates, the number of neighbors, and the transmission power in order to calculate the radio transmission range. (2) Collision Assessment, in this phase nodes use Eq. (37) to determine the collision threshold using the information provided in the RTB control messages. Then, nodes use Eq. (38) to determine whether they are allowed to retransmit or not. Enabled nodes are those with a collision probability lower than the calculated collision threshold. These enabled nodes go to the third phase, the CTB transmission phase in which nodes send back to the source node a Clear-to-Broadcast (CTB) packet. This packet is only used to inform the source node about the potential forwarders. Finally, CAREFOR algorithm also uses silencing mechanism based on the forwarding probability (36).

In [104] (GOSSIP_DIR2), the authors used directional antennas and Euclidean distance. The additional coverage area is calculated independently for each sector. Thus, a different forwarding probability is assigned to each sector in order to construct the probability vector $p=\left(p_{1}, \ldots, p_{S_{c}}\right)$ where $S_{c}$ is the number of sectors of the directional antennas. The distance between the sender $S$ and the receiver $R$ and the Angle of Arrival (AOA). In [104] the authors proposed an estimation technique to calculate the additional coverage area in each sector. Using the proposed estimation technique, the forwarding probability for each section can be calculated as,

$p_{i}=\max \left(0,1-\left(\frac{d_{i}^{\prime}}{r}\right)^{2}\right)$

where $d^{\prime}$ is the estimated Euclidean distance.

\section{(c) Hop count-based:}

The hop count is used in [42], where the authors proposed GOSSIP4, a scheme based on the performance of the hybrid routing protocol ZRP [43]. The nodes act reactively or proactively depending on the distance from the source node. Such distance is measured in number of hops. Nodes maintain a zone that is composed of nodes located within $\Lambda$ hops from the source node. Nodes perform proactively inside these zones, so as to trigger the exchange of neighboring information each time a change occurs in the zone. On the other hand, nodes located out of the zone are called peripheral nodes and they perform reactively. In [42] the authors defined GOSSIP4(p,k, $\left.\mathrm{k}^{\prime}\right)$, which performs similar to GOSSIP1 $(\mathrm{p}, \mathrm{k})$ but the nodes have a zone of radius $k^{\prime}$. In summary, this scheme performs as $\operatorname{GOSSIP} 1(\mathrm{p}, \mathrm{k})$ for those nodes located out of the zone, and as simple flooding for those nodes inside the zone.

\section{(d) Hints-based:}

A quite different type of broadcast scheme is proposed in $[17,16,19]$ where, the authors presented polarized GOSSIP a scheme based on the relative positions between a given node and the destination node. A node forwards an incoming packet with probability $p_{h}$ if it is closer to the destination than the previous node, otherwise the forwarding probability is $p_{l}$. The relative distance between a node and the destination node is estimated by "hints". The hint of a node $i$ with respect to the destination node $j$ is 0 if the 
two nodes are one hop neighbors, otherwise $h_{i n t} t_{i, j}=\frac{\Delta T_{i, j}}{d u r_{i j}}$. Here, $\Delta T_{i, j}$ is the time elapsed since the last time $i$ and $j$ were neighbors and $d u r_{i, j}$ is the last link's duration between $i$ and $j$. The correlation between hint $_{i, j}$ and the Euclidean distance has been empirically studied [19]. In polarized GOSSIP a node sends heartbeat packets to its neighbors (similar to hello packets) every $\Delta T_{S}$ and uses a vector of time information $\mathrm{VH}_{i}$.

3.2.1.2.2. Location-based schemes. In [64] (regional GOSSIP), nodes have to use some location service to collect positioning data. The main objective behind Regional GOSSIP is to limit the number of retransmissions, and only the nodes that are in certain regions linking the source and the destination nodes are allowed to forward the incoming messages. The forwarding regions defined by the nodes are elliptical areas using the source node and destination node as the foci, see Fig. 10.

Furthermore, the forwarding probability is also adjusted dynamically by using the density of nodes within these ellipses. To carry out the propagation of messages, the geometrical information of the source node and the destination node and also the current route is piggybacked on the broadcast packets. Whenever a node $v$ receives a new message it has to check whether it is inside the ellipse defined. If the nodes is inside the ellipse, it uses the following forwarding probability.

In [64] the authors derived the forwarding probability in the regional gossiping area as,

$p \simeq \frac{\ln \left(N \pi l^{2} / \tilde{4}\right)}{N \pi^{2} \tilde{l}^{2} \tilde{r}^{2} / 4}$

where $l$ is the ellipse factor, $\hat{l}^{2}=l \sqrt{l^{2}-1}, \tilde{r}^{2}=\frac{r}{a}$, and $a$ is the length of site in a square scenario. The simulation results showed that the number of retransmissions generated using this scheme is less than the simple GOSSIP. In fact, up to $94 \%$ of messages can be saved compared to simple GOSSIP. However, this approach presents several disadvantages: (1) the nodes need to develop a location service in order to collect positioning data, and (2) this location service adds overhead to the network so care must be taken in order not to worsen the performance of the network. In addition, the forwarding probability expression (42) depends on the total number of nodes in the network, which is a global parameter that in most cases is unknown to the nodes.

A summary of the main parameters of the reviewed distance-based schemes is presented in Table 5.

3.2.1.3. Speed-based schemes. The speed of nodes is specially relevant in VANETs where the speed of nodes is much higher than in other types of ad hoc networks such as MANETs or WSNs.

In [79] (SAPF), the authors proposed estimating the network's density in VANETs based on the node's speed. In SAPF the forwarding probability is adjusted as follows:

$p=0.0557 v-0.033$

where $v$ is the vehicle's speed. Two speed thresholds are defined $v_{l}$ and $v_{h}$. The authors indicated that if $v>v_{h}$, it is impossible to estimate the vehicle's density. On the other hand, if $v<v_{l}$, the network has almost reached its capacity and so the probability can be constant.

3.2.1.4. Self-pruning based schemes. In self-pruning schemes, the main objective is to cover the set of 2 hops neighbors of a given node. In this type of probabilistic schemes, the forwarding probability is adjusted by considering how this set of 2 hops neighbors is covered.

In [4] (DPR), the authors used a self-pruning mechanism to calculate the forwarding probability that is adjusted according to the number of neighbors uncovered. The following expression is used:

$p=\frac{n_{b}-n_{\text {cov }}}{n_{a v g}}$ if $\quad n_{b} \leqslant n_{a v g}$

$p=\frac{n_{b}-n_{\text {cov }}}{n_{b}}$ if $n_{b}>n_{a v g}$

where $n_{c o v}$ is the number of neighbors which have already been covered by previous copies of the same broadcast packet. The authors estimate the average number of neighbors using the following expression,

$n_{\text {avg }}=\frac{(N-1) \pi r^{2}}{A_{n e t}} \alpha$

where $\alpha$ is estimated using massive number of simulations [4].

In NCPR [129], the forwarding probability is calculated as a function of two metrics, (a) the additional coverage ratio, and (b) the connectivity factor. The additional coverage factor is calculated as follows:

$R_{a}(i)=\frac{|U(i)|}{N_{b}(i)}$

where $U(i)$ is the uncovered neighbors of node $i$ (selfpruning mechanism), which can be calculated using the following expression,

$U(i)=N_{b}(i)-\left[N_{b}(i) \cap N_{b}(i-1)\right]-\{i-1\}$

Notice that the additional coverage metric measures the set of new nodes that is covered if node $i$ forwards the packet. On the other hand, the connectivity factor is expressed as follows:

$F_{c}(i)=\frac{N_{c}}{N_{b(i)}}$

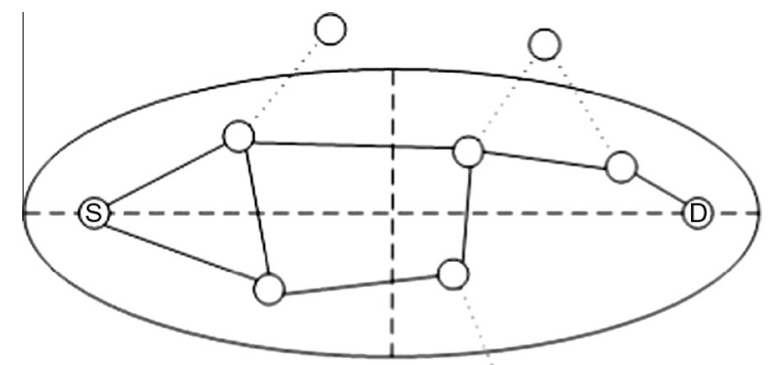

Fig. 10. Forwarding area in regional GOSSIP. 
Table 5

Adaptive non-counter and area based-probabilistic schemes.

\begin{tabular}{|c|c|c|c|c|c|}
\hline Scheme & Subtype & Forwarding probability & Collected data & Adjusted parameters & Application \\
\hline BNR [33] & Density & $p=\frac{A-\alpha}{M^{\sigma}} \mu^{\sigma}+\alpha$ & $\mu$ & $A, \alpha, \sigma$ & MANET \\
\hline B-Overlap [103] & Density & $p=\frac{D R}{N N O A}$ & $n_{b}$ & $D R$ & MANET \\
\hline PPR [103] & Density & $p=\frac{D R}{\left(1-\varepsilon^{2}\right)} L N D$ & $n_{b}$ & $D R$ and $\varepsilon$ & MANET \\
\hline Jaccard distance [98] & Density & $p=J d$ & $J d$ & & MANET \\
\hline Weighted p-persistence [115] & Euclidean and RSS & $p=\frac{d_{i j}}{r}$ & $d$ or $R S S$ & - & VANET \\
\hline NPPB [130] & Euclidean & $p=\left(\frac{d_{i j}}{r}\right)^{k}$ & $d$ & $k$ & VANET \\
\hline ACPF [69] & Euclidean and RSS & 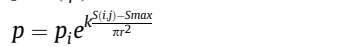 & $d$ or RSS & $k$ and $p_{i}$ & MANET \\
\hline DPBSC [111] & RSS & $\begin{array}{lll}p=\frac{S(i, j)}{S_{\max }(i, j)} & \text { if } & 0<d<r \\
0 & \text { Otherwise } & \end{array}$ & RSS & - & MANET \\
\hline IF [89] and CIF [29] & Euclidean and RSS & $p=\left(1-F_{X}(z-d)\right)^{\frac{1}{c}}$ & $d$ and $\varphi$ & $k$ & VANET \\
\hline CAREFOR [77] & Euclidean & $p=e^{-\frac{\varphi_{s}\left(r-d_{i, j}\right)}{k} r_{i}}$ & $d$ and $\varphi$ & $k$ & VANET \\
\hline GOSSIP_DIR2 [104] & Euclidean & $p_{i}=\max \left(0,1-\left(\frac{d}{r}\right)^{2}\right)$ & $\mathrm{d}$ and $\vartheta$ & - & MANET \\
\hline GOSSIP4 & Hop & $\begin{array}{lll}p=p_{i} & \text { if } & \text { hops }<\Lambda \\
\text { else } & p=1 & \end{array}$ & hops & $\Lambda$ & MANET \\
\hline Polarized GOSSIP [17] & Hints & $\begin{array}{ll}p=p_{h} & \text { if } \\
\text { else } & p=p_{l}\end{array}$ hint $_{i, j_{i}}>$ hint $_{i, j_{i-1}}$ & $V H_{i}[j]$ & $p_{h}$ and $p_{l}$ & MANET \\
\hline
\end{tabular}

where $N_{c}=5.1774 \log (N)$. The value of $N_{c}$ is derived in [117], in this paper the authors estimated that if each node is connected to more than $5.1774 \log (N)$ of its nearest neighbors, then the probability of the network being connected approaches 1 , as the number of nodes in the network increases. Although this metric is suitable to ensure connectivity in the network, it is impractical in most cases, since it relies on knowing the total number of nodes in the network, which is a global network parameter. Finally, the forwarding probability is given by:

$p(i)=F_{c}(i) \cdot R_{a}(i)$

According to Eqs. (45) and (47) the value of forwarding probability given by (48) can be higher than 1 . In order to avoid such condition, the maximum probability is fixed to 1. In addition, the authors proposed to calculate the rebroadcast delay based on the number of common neighbors of a given node with its previous node in the communication path. They predicted that if the number of shared neighbors is higher, the forwarding delay should be lower, and therefore, more neighbors can be reached. The rebroadcast delay is calculated as follows:

$T_{p}(i)=$ MaxDelay $\times t_{p}(i)$

where $t_{p}(i)$ is the delay ratio of node $i$, which is calculated using the following expression,

$t_{p}(i)=1-\frac{\left|N_{b}(i-1)-N_{b}(i)\right|}{\left|N_{b}(i-1)\right|}$

where $N_{b}(i)$ is the set of neighbors of node $i$.

A summary of the described self-pruning schemes is presented in Table 6 .

3.2.1.5. Energy-based schemes. The following schemes are focused on reducing the power consumption of the broadcast schemes so energy-based parameters are used to adjust the forwarding probability. In general power consumption is an important issue in wireless ad hoc networks since nodes are normally powered by batteries.

In [83] a simple probabilistic scheme based on the node's energy level is proposed (ECG). The authors defined an energy level threshold $E_{t h}$. Whenever a given node receives a broadcast packet, it checks its energy level and if it is lower than $E_{\text {th }}$, it will not forward the incoming packet. Otherwise, the node forwards the packet with a probability $p=0.75$. Moreover, if the node has only one neighbor, the forwarding probability is 1 .

In [50] (EAG), the residual energy of nodes is used in [50] as the key parameter to adjust the node's forwarding probability. The forwarding probability is calculated as follows:

$p=0.6 \varphi_{i} \quad$ if $\quad n_{b}>5$

else $p=1$

where $\varphi_{i}$ is the residual energy of a node $i$ and the constant value 0.6 is fixed to ensure certain level of reachability. Furthermore, a threshold $n_{c}=5$ is defined.

In [84] (EBG), nodes use their battery levels to adjust the retransmission probability as follows:

$p=\frac{E(\%)}{100}$

where $E(\%)$ is the percentage of remaining energy. As a consequence, nodes with higher battery level have higher forwarding probability.

In NEBG scheme [93] the energy levels of nodes located in the node's neighborhood are considered to determine the forwarding probability. This scheme adapts the forwarding probability to the neighborhood energy conditions. On receiving a new broadcast packet a node $i$ calculates the forwarding probability according to the following expression:

$p=\frac{E_{i}-E_{\min }}{E_{\max }-E_{\min }}$ 
Table 6

Adaptive non-counter-based self-pruning based probabilistic schemes.

\begin{tabular}{llll}
\hline Scheme & Forwarding probability & Collected data & Adjusted parameters \\
\hline DPR [4] & $\begin{array}{l}p=\frac{n_{b}-n_{\text {cov }}}{n_{a v g}} \text { if } n_{b}<n_{\text {avg }} \\
p=\frac{n_{b}-n_{c o v}}{n_{b}}\end{array}$ if $n_{b}<n_{\text {avg }}$ & $n_{b}$ and $N$ & Application \\
NCPR [129] & $p=F_{c} R_{a}$ & $n_{b}$ and $N$ & MANET \\
\hline
\end{tabular}

where $E_{\max }$ and $E_{\min }$ are the maximum and minimum energy levels in the node's neighborhood. This is more advantageous in scenarios where the nodes can have similar values of energy level.

A summary of the reviewed energy-based schemes is presented in Table 7.

3.2.1.6. Based on artificial intelligence. The following schemes use artificial intelligence to adjust the forwarding probability of nodes. In [2] a genetic algorithm is used to calculate the forwarding probability. Other parameters such as number of repeats that a given node retransmits an incoming packet, delay between repeats, and Time To Live (TTL) for a packet, are taken into consideration to evaluate the fitness function. The parameters to be minimized are number of collisions, propagation time, and number of retransmissions. The authors evaluated the proposed approach under different densities in VANET scenarios.

In [94], a multi-objective genetic algorithm is used to optimize probabilistic broadcast in disaster scenarios. The main difference from the single objective optimization problem is that several output metrics are considered to obtain the set of non dominated solutions (Pareto front).

\subsubsection{Adaptive counter-based schemes}

In counter-based schemes, nodes use the number of received copies of a given broadcast packet as a metric to determine the state of the broadcast process in their vicinities. Thus, the main idea is to avoid the die out problem of the broadcast process. As shown in Fig. 2, other factors can also be combined to adjust the forwarding probability such as density metrics in density-based schemes, the relative distance in distance-based schemes, self-pruning mechanisms, and the energy of nodes.

3.2.2.1. Density-based schemes. Similar to the non counterbased schemes, the adaptive counter-based schemes based on density can be further divided into different categories (Fig. 11) such as pure counter-based, node's degree, density thresholds, and color-based. The pure schemes use only the number of copies received as the factor to determine the forwarding probability. In node's degreebased schemes, the number of received copies is combined with the number of node's neighbors. In addition, density thresholds can also be defined based on the number of neighbors and the number of received copies. Finally the color-based scheme is a similar approach to the pure counter-based schemes but considering a color-field as the main parameter to adjust the forwarding probability.

\section{(a) Pure:}

GOSSIP3 is proposed in [42,41], which is the basic counter-based scheme. Normally, it is very difficult to know if the Gossip is dying out, however this occurrence could be predicted based on the number of received copies of a packet that a node receives from its neighbors. A node with $n_{b}$ neighbors and a forwarding probability $\mathrm{p}$ should receive $m=n_{b} p$ copies of a given packet. If a given node receives fewer than $m$ copies, it is reasonable to say that the die out problem is happening. This approach is called GOS$\operatorname{SIP} 3(\mathrm{p}, \mathrm{k}, \mathrm{m})$ and it requires the node to count the number of copies for a given packet. If a node could not forward a packet due to the probabilistic decision, then the node waits for a period of time counting the number of copies, and if this number of copies is higher than $m$, the node will decide that it is not necessary to retransmit the packet. Otherwise, the node will retransmit the packet with $p=1$ after the waiting time. Two important parameters should be correctly adjusted, (1) the threshold value of the number of copies $h$ and (2) how long should the nodes wait before retransmitting the packet. This time is random and is known as the Random Assessment Delay (RAD). In [42], the authors estimated that $h$ should be equal to 1 and the waiting time RAD should be quite small in order not to delay the communications. The main disadvantages of GOSSIP3 are (1) a delay in communications due to the RAD delay and (2) the isolated node problem, which is represented in Fig. 12. The node $\mathrm{H}$ can only be reached via node $F$, but node $F$ will forward the incoming packet with a low probability since it has many neighbors (three neighbors). As a consequence, the node $H$ could not be reached.

Table 7

Dynamic energy based probabilistic schemes.

\begin{tabular}{|c|c|c|c|c|}
\hline Scheme & Forwarding probability & Collected data & Adjusted parameters & Application \\
\hline ECG [83] & $\begin{array}{lll}p=0.75 & \text { if } \\
\text { else } & p=0\end{array} \quad E>E_{\text {th }}$ & $E$ & $E_{t_{h}}$ & MANET \\
\hline EAG [50] & $\begin{array}{lll}p=0.2 \varphi_{i}+0.6 & \text { if } & n_{b}>5 \\
\text { else } & p=0 & \end{array}$ & $n_{b}$ and $\varphi$ & $n_{c}$ & MANET \\
\hline EBG [84] & $p=\frac{E(\%)}{100}$ & $E(\%)$ & - & MANET \\
\hline NEBG [93] & $p=\frac{E-E_{\min }}{E_{\max }-E_{\min }}$ & $E$ & - & MANET \\
\hline
\end{tabular}




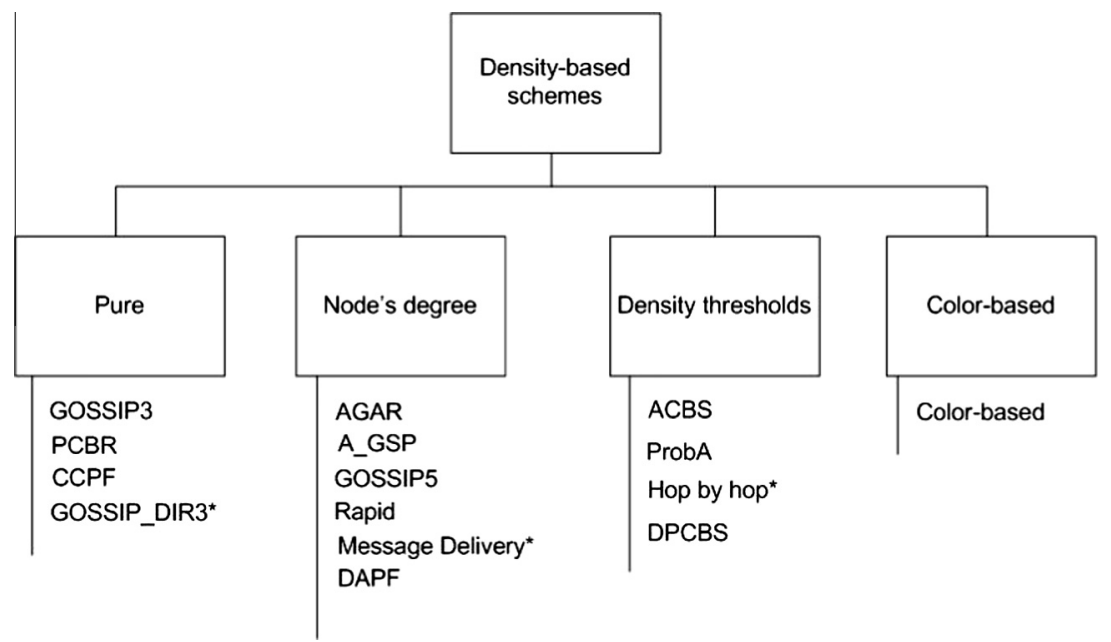

Fig. 11. Classification of density-based schemes.

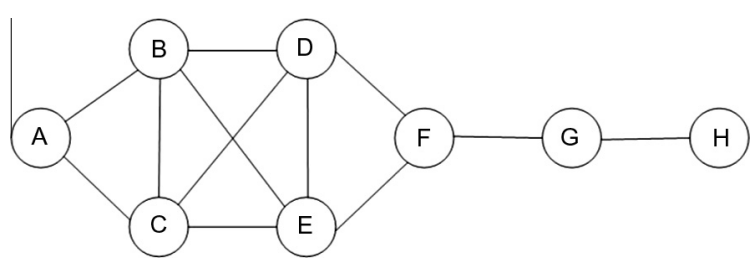

Fig. 12. The isolated node problem.

Another similar version of GOSSIP3 is PCBR proposed in [76]. However, there are two main differences of PCBR from GOSSIP3: (1) GOSSIP3 employs simple flooding during the first $\mathrm{k}$ hops, whereas PCBR always uses probabilistic broadcast and (2) In PCBR the retransmissions are always delayed by RAD timer, in contrast, GOSSIP3 only delays retransmissions as long as the probabilistic operation decides not to retransmit. Fig. 13 illustrates the latter.

In CCPF scheme [69], the authors used an exponential function based on the number of received copies to adjust the forwarding probability. The forwarding probability is calculated as,

$p=p_{i} \cdot e^{-k m}$

where $p_{i}$ is a constant probability to ensure connectivity and $k$ is a parameter to control the maximum and minimum probabilities. There are two main differences with respect to GOSSIP3: (1) Nodes do not attempt to retransmit before the waiting time RAD, consequently, the retransmissions are always delayed and (2) there is no defined threshold for the number of copies $m$ so nodes always retransmit with a forwarding probability determined by (55) after waiting for a period of time RAD.

In GOSSIP_DIR3 [104], the authors combine the probabilistic counter-based scheme with the use of directional antennas. A different counter threshold $h_{j}$ is defined for each sector $j$ of the directional antenna. The values of the probability vector $\vec{p}=\left(p_{1}, \ldots, p_{S_{c}}\right)$ are calculated as follows: $p_{j}=\min \left(\frac{p_{i} S_{c}}{S_{c}-1}\left(1-\frac{h_{j}}{\sum_{j=1}^{S_{c}} h_{j}}\right), 1\right)$

where $S_{c}$ is the number of sectors, $j=1, \ldots, S_{c}$, and $p_{i}$ is the initial forwarding probability as defined in GOSSIP3 [42].

(b) Node's degree:

The following schemes combine the counter-based mechanism with the node's number of neighbors in order to adjust the forwarding probability.

In AGAR scheme [105] a node forwards the incoming packet with a probability given by (57) whenever its receives fewer than $h$ messages. So instead of using $p=1$, the nodes will forward the packet with a probability,

$p=\frac{p_{i}}{n_{b}+1}$

As a result, the probability will depend on the node's neighbors resulting in a reduction in the number of broadcast messages.

In A_GSP scheme [63], the authors proposed a very similar version of AGAR [105], where they proposed to use the following expression (58) instead of (57),

$p=\frac{p_{i}}{n_{b}}$

In GOSSIP5 [22], the authors proposed a similar scheme to GOSSIP3. But, GOSSIP5 exhibits two basic differences from GOSSP3. On one hand, the counter threshold is fixed to the node's number of neighbors so $h=n_{b}$. On the other hand, nodes always wait a fixed RAD before trying to retransmit the incoming packet as in PCBR.

In [54] (message delivery), the authors categorized the nodes into two categories according to the number of neighbors. If a node has fewer than $n_{c}$ neighbors it is considered as a $\beta$ node, otherwise as an $\alpha$ node. At the same time the broadcast packets are also categorized as $\alpha$ and $\beta$ packets. A packet sent by an $\alpha$ node is categorized as an $\alpha$ packet. If the packet is sent by a $\beta$ node it is considered 

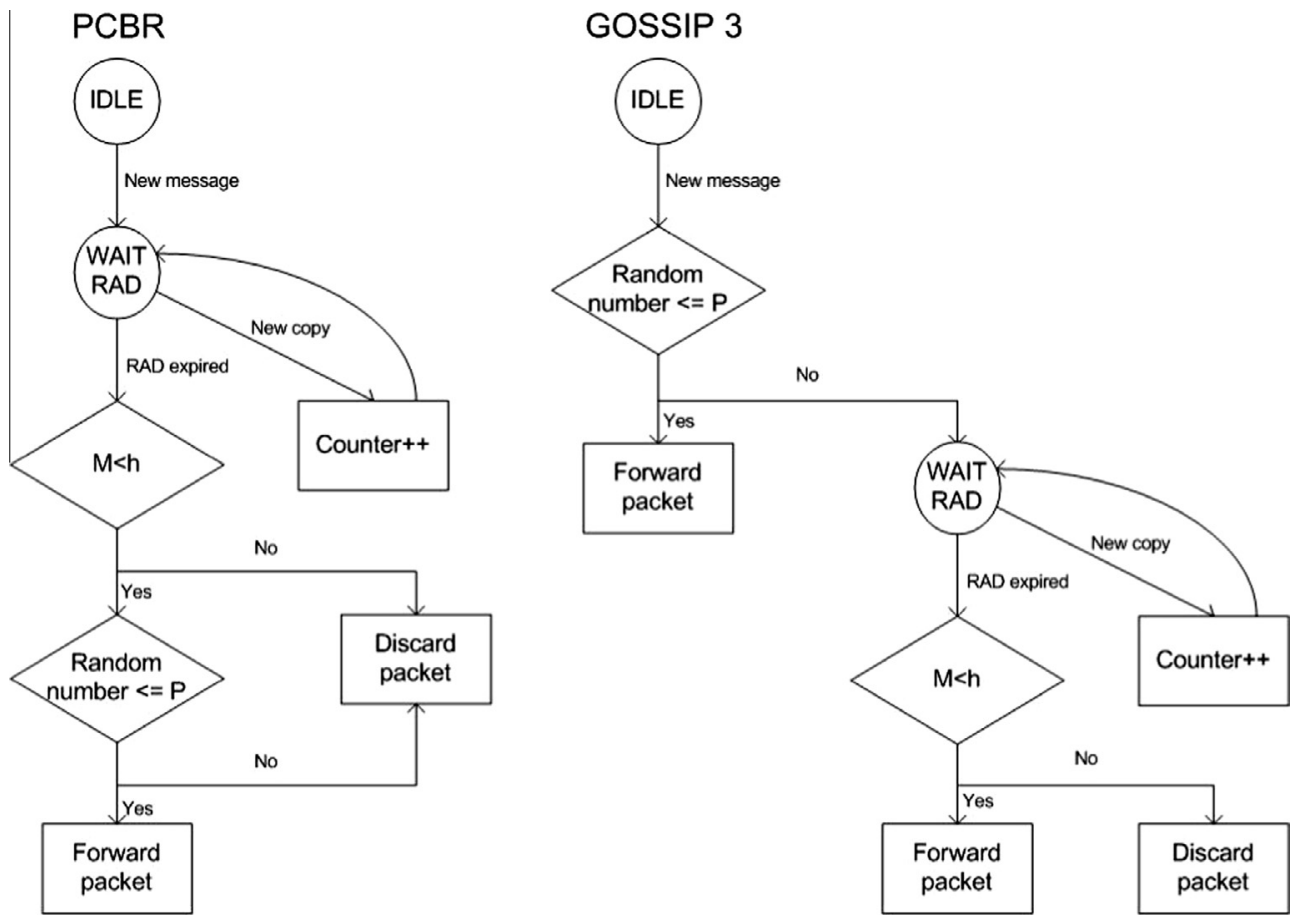

Fig. 13. Difference between GOSSIP3 and PCBR.

as a $\beta$ packet. Whenever a $\beta$ node receives a $\beta$ packet, it will be always forwarded with $p=1$. Otherwise, the nodes wait for a time $t_{1}$ before broadcasting the packet with a probability,

$p=\frac{1}{m n_{c}}$

In [85](DAPF), the authors presented a multi phase approach. At each $\Delta t$ (the time interval between phases) the nodes adjust their forwarding probability for the next phase using the following expression:

$p_{j}=\frac{1}{\left(n_{b}+1\right)-\frac{j}{3} n_{b}}$

where $j=0,1,2,3$ is the node's phase, so up to four phases are defined. Initially, each node tries to forward the incoming packet. If the node does not forward the packet, it will start to count the number of received duplicated packets $m$ and it will wait for a RAD time. After the expiry of RAD, the node passes to the next phase and adjusts its forwarding probability according to (60). In each phase $h=2$ to decide whether the node must try to transmit or not. In phase 3, the nodes always forward with $p=1$ to ensure higher reachability.

In RAPID scheme [36,37], deterministic and probabilistic broadcast are combined to ensure successful delivery of messages in ad hoc networks [36,37]. First, the authors proposed that the forwarding probability in probabilistic schemes should be inversely proportional to the number of neighbors of a given node. They derived the following expression; $p=\min \left(1, \frac{\beta}{n_{b}}\right)$

where $\beta$ is a parameter called the reliability factor. The authors stated that $\beta=3.5$ is a good tradeoff between the number of retransmissions and the reliability level. Moreover, the authors implemented a counter-based version of their original scheme in order to reduce the number of redundant packets. In addition, nodes periodically broadcast to their neighbors the header of messages received from other nodes in order to avoid nodes missing packets. This technique is called Lazy gossip [52] and is deterministic. it has some shortcomings such as an increment of overhead and latency. However, the authors stated that this is the only way to guarantee $100 \%$ reliability. Finally, RAPID also uses jitter to avoid collisions so nodes rebroadcast after waiting RAD time.

(c) Density thresholds:

As with non-counter based schemes, density thresholds are also used in counter-based schemes. Notice that in this case the density thresholds are based on the number of received copies instead of the number of neighbors as in non counter-based schemes.

In ACBS scheme [74], the forwarding operation is not suppressed when a density threshold value of $h$ is reached, instead a low value of forwarding probability is used. The forwarding operation is then controlled by the following expression:

$$
\begin{array}{ll}
p=p_{h} & \text { if } \quad m<h \\
\text { else } & p=p_{l}
\end{array}
$$


In ProbA scheme [66] multiple density thresholds is proposed. The retransmission probability is calculated as follows:

$$
\begin{array}{lll}
p=p_{1} & \text { if } & m<h_{1} \\
p=p_{2} & \text { if } & h_{1}<m<h_{2} \\
p=p 3 & \text { if } & h_{2}<m<h_{3} \\
\cdots & \ldots & \cdots \\
p=p_{n} & \text { if } & h_{n-1}<m<h_{n}
\end{array}
$$

where $p_{1}, p_{2}, p_{3}, \ldots, p_{n}$ are the forwarding probabilities, and $h_{1}, h_{2}, h_{3}, \ldots, h_{n}$ are the counter-based thresholds. The authors evaluated this scheme with three threshold values $h_{1}=1, h_{2}=2$, and $h_{3}=3$ and the forwarding probabilities were $p_{1}=0.9, p_{2}=0.5$, and $p_{3}=0.1$.

In [128] (hop by hop), the authors proposed a probabilistic scheme that adapts the forwarding probability hop by hop as a broadcast packet goes through the nodes in a network. If the number of copies is lower than $h$, the value of $p$ is incremented by a fixed amount $p_{\Delta_{-}}$. In contrast, if the number of copies is higher than $h$, the value of $p$ is decremented by $p_{\Delta+}$. The authors also defined two limits for the value of $p$, these values are $p_{l}$ and $p_{h}$, where $p_{h}>p_{l}$. So the forwarding probability is calculated at each node as follows:

$$
\begin{array}{lll}
p=p_{i}-p_{\Delta+} & \text { if } & m<h \\
p=p_{i}+p_{\Delta-} & \text { if } & m>h \\
p=p_{l} & \text { if } & p<p_{l} \\
p=p_{h} & \text { if } & p>p_{h}
\end{array}
$$

At every interval $t$, the nodes compute $m$ in order to determine the retransmission probability. Unlike the classical counter based implementation, here the nodes evaluate $m$ at each interval $t$ and the nodes make the decision of forwarding the incoming packet immediately after receiving a packet. Therefore the broadcast latency is lower than that of the classical counter based schemes. However, the authors did not describe how they arrived at the optimum value of $t$. They used the results shown in [108] to select $h=6$ as the threshold value in order to save rebroadcast packets. Another important parameter to be adjusted is the initial probability $p_{i}$, a low value of $p_{i}$ may result in a low reachability. While a high value of $p_{i}$ may increase the number of redundant packets. The authors determined the initial probability on the basis of average number of neighbors of a given node $n_{a v g}$ and it can be calculated using (6). The initial forwarding probability is determined as follows:

$$
\begin{array}{lll}
p_{i}=1 & \text { if } & \frac{6}{n_{\text {avg }}} \geqslant 1 \\
p_{i}=\frac{6}{n_{\text {avg }}} & \text { if } & 0<\frac{6}{n_{\text {avg }}}<1 \\
p_{i}=0 & \text { if } & \frac{6}{n_{\text {avg }}} \leq 0
\end{array}
$$

In [75] (DPCBS), the authors proposed a counter-based scheme with a piecewise probability function based on the number of copies received. The main difference from previous schemes is that DPCBS uses an exponential probability function that is defined as follows:

$$
\begin{array}{ll}
p_{1}=e^{-\left(\frac{m}{h}\right)} & \text { if } \quad m<h \\
p_{2}=e^{-\left(\frac{m+1}{h}\right)} & \text { Otherwise }
\end{array}
$$

Nodes in DPCBS perform as in PCBR so they always wait RAD. The authors used a threshold $h=3$ to evaluate the performance of DPCBS.

\section{(d) Color-based:}

A variation counter-based scheme is proposed in [55] (color-based broadcasting). In this scheme, every broadcast message has a color-field [55]. The condition to be satisfied at the expiration time is similar to the original counterbased scheme so the number of colors of broadcast messages overhead must be less than a threshold. If this condition is satisfied the message will be retransmitted with a new color assigned to its color-field. The authors argue that the color-based schemes create backbones richer than the counter-based schemes in terms of robustness against node's failures since color-based schemes create mesh-like backbones. In fact, the authors proved that for any backbone generated by a counter-based scheme there exits a backbone that can result from the color-based scheme with the same threshold $m$, which contains the counterbased backbone as a subset. The authors analyzed and compared the $m=2$ case, called Red-Blue broadcast, with the same counter-based approach. The simulation results showed that both approaches perform similarly in terms of reachability, while color-based scheme produces less number of rebroadcasts.

Tables 8 and 9 include the main parameters of the reviewed density-based schemes.

\subsubsection{Distance-based schemes. In distance-based} schemes, nodes combine the counter-based mechanism with the relative distance between two nodes in order to determine the forwarding probability. Notice that although in counter-based schemes we have not made the same classification of distance-based schemes as it is done in the non counter-based case, the same approximations used in the non counter-based case are also applicable to the counter-based case.

In [56] (DDAPF), the authors proposed calculating the relative distance between two nodes as follows:

$d_{r}=\frac{d_{T, R}}{r_{R}}$

where $r_{T}$ is the transmitter respectively and $d_{T, R}$ is the Euclidean distance between the transmitter and the receiver. Notice that this is a relative measurement. The authors also proposed to use the received power to estimate the Euclidean distance. In DDAPF, the forwarding probability is calculated as follows:

$$
\begin{array}{cc}
p=p_{h} & \text { if } \quad m=1 \\
\text { otherwise } & p=\min \left(p_{l,}\left(\frac{e-e^{\left(1-\frac{d_{r}}{d_{\max }}\right)}}{e-1}\right)^{k}\right)
\end{array}
$$

where $d_{\max }$ is the maximum distance, $k$ a shaping parameter, and $p_{h}$ and $p_{l}$ are the maximum and minimum for- 
Table 8

Adaptive counter based and density based probabilistic schemes I.

\begin{tabular}{|c|c|c|c|c|c|}
\hline Scheme & Subtype & Forwarding probability & $\begin{array}{l}\text { Collected } \\
\text { data }\end{array}$ & $\begin{array}{l}\text { Adjusted } \\
\text { parameters }\end{array}$ & Application \\
\hline GOSSIP3 [42] & Pure & $\begin{array}{llll}p=p_{i} & \text { if } \quad t<\tau \quad \\
\text { else } & p=1 \quad \text { if } \quad m<h \\
\text { else } & p=0 & & \end{array}$ & $m$ & $R A D, h, p_{i}$ and $k$ & MANET \\
\hline PCBR [76] & Pure & $\begin{array}{ll}p=p_{i} & \text { if } \\
\text { else } & p=0\end{array} \quad m<h$ & $m$ & $R A D, h, p_{i}$ and $k$ & MANET \\
\hline CCPF [69] & Pure & $p=p_{i} \cdot e^{-k m} \quad t=\tau$ & $m$ & $R A D, p_{i}$ and $k$ & MANET \\
\hline $\begin{array}{l}\text { GOSSIP_DIR3 } \\
{[104]}\end{array}$ & Pure & $p_{j}=\min \left(\frac{p_{i} S_{c}}{S_{c}-1}\left(1-\frac{h_{j}}{\sum_{j=1}^{S_{c}} h_{j}}\right), 1\right)$ & $m$ & $p_{i}$ and $h_{j}$ & MANET \\
\hline AGAR [105] & Degree & $\begin{array}{llll}p=p_{i} & \text { if } & t<\tau & \\
\text { else } & p=\frac{p_{i}}{n_{b}+1} & \text { if } \quad m<h \\
\text { else } & p=0 & & \end{array}$ & $m$ and $n_{b}$ & $R A D, h, p_{i}$ and $k$ & MANET \\
\hline A_GSP [63] & Degree & $\begin{array}{llll}p=p_{i} & \text { if } \quad t<\tau \quad \\
\text { else } & p=\frac{p_{i}}{n_{b}} \quad \text { if } \quad m<h \\
\text { else } & p=0 & & \end{array}$ & $m$ and $n_{b}$ & $R A D, h, p_{i}$ and $k$ & WSN \\
\hline Rapid & Degree & $p=\min \left(1, \frac{\beta}{n_{b}}\right)$ & $m$ and $n_{b}$ & $R A D$ and $\beta$ & MANET \\
\hline $\begin{array}{l}\text { Message Delivery } \\
\quad[54]\end{array}$ & Degree & $\begin{array}{l}p=\frac{1}{m n_{c}} \text { at } t=\tau \text { if current node and previous node both are } \alpha \text {, } \\
\text { otherwise } p=1\end{array}$ & $m$ and $n_{b}$ & $R A D$ and $n_{c}$ & MANET \\
\hline DAPF [85] & Degree & $p_{j}=\frac{1}{\left(n_{b}+1\right)-\frac{j}{3} n_{b}}$ & $m$ and $n_{b}$ & $R A D$ & MANET \\
\hline
\end{tabular}

warding probabilities. In addition, a back off delay time based on distance is also proposed. The back off delay is calculated as follows:

Delay $=$ delay $_{\max }\left(\frac{e-e^{\left(\frac{d_{r}}{d_{\max }}\right)}}{e-1}\right)^{k}$

where delay $_{\max }$ is the maximum delay a packet can experience at each node.

In ACCPF scheme [69], the authors combined the features of ACPF (non counter-based that uses distance) and CCPF (pure counter-based) in order to get benefits from both schemes. In ACCPF, nodes take into account both the Euclidean distance and the number of copies received in order to compute the forwarding probability (68). In this scheme, each node maintains the number of copies $m$, and the smallest additional coverage area $S_{\min }$ for each broadcast packet $m$. After a waiting period of time $R A D$, the nodes retransmit with a probability,

$p=p_{i} e^{k_{1} \frac{S \min (m)-S_{\max }}{\pi r^{2}}} e^{-k_{2} m}$

where $S_{\max }=0.61 \pi r^{2}, r$ is the node's transmission range, $k_{1}$ and $k_{2}$ are two constants used to control the forwarding probability, and $S_{\min }$ is updated every time a node receives a new copy of $m$.

In Discount scheme [49], the perimeter nodes are preferred. A distance threshold $D_{t h}$ determines which nodes are perimeter nodes and which are inner nodes. If a given node is located in the area defined by $D_{t h}$ it is considered as an inner node, for instance, node $\mathrm{j}$ in Fig. 14. Otherwise, the node will be considered as a perimeter node, see node $k$ in Fig. 14. An inner counter threshold $I C_{t h}$ is defined in order to limit the forwarding operation of inner nodes. If the number of copies $m$ is higher than $I C_{t h}$, the inner node will not forward the incoming packet. In summary, the objective consists of reducing the number of rebroadcasts made by the inner nodes.

An extension of Discount scheme is presented in [49] (Discount-RS). The authors identified that discount scheme could lead to a large number of redundant packets if the source node has many border nodes. In order to avoid such redundancy, the authors incorporated counter-based operation to the border nodes. If a border node receives a copy of a packet from another border node, it will not forward the incoming packet.

Table 10 includes the main parameters of the reviewed distance-based schemes.

3.2.2.3. Self-pruning based schemes. In NCPF a self-pruning mechanism is added to ACCPF (counter-based scheme that uses relative distance) algorithm [69]. Nodes do not rebroadcast if their entire transmission areas have already been covered by other nodes. To insert this self-pruning mechanism in ACCPF, the broadcast messages have to

Table 9

Adaptive counter based and density based probabilistic schemes II.

\begin{tabular}{|c|c|c|c|c|c|c|c|c|}
\hline Scheme & Subtype & Forward & ling pr & robability & & Collected data & Adjusted parameters & Application \\
\hline ACBS [74] & Thresholds & $\begin{array}{l}p=p_{h} \\
\text { else }\end{array}$ & $\begin{array}{l}\text { if } \\
p=\end{array}$ & $\begin{array}{l}m<h \\
p_{l}\end{array}$ & & $m$ & $R A D, h, p_{l}, p_{h}$ and $k$ & MANET \\
\hline ProbA [67] & Thresholds & (63) & & & & $m$ & $R A D, p_{1}, \ldots, p_{n}$ and $h_{1}, \ldots, h_{n}$ & MANET \\
\hline Hop by Hop [128] & Thresholds & (64) & & & & & $R A D, p_{i}, p_{\Delta+}, p_{l}, h, p_{\mu}$, and $p_{-}$ & MANET \\
\hline DPCBS [75] & Thresholds & $\begin{array}{l}p_{1}=e^{-} \\
p_{2}=e^{-}\end{array}$ & & $\begin{array}{l}\text { if } \\
\text { Otherwise }\end{array}$ & $m<h$ & $m$ & $h$ & MANET \\
\hline
\end{tabular}




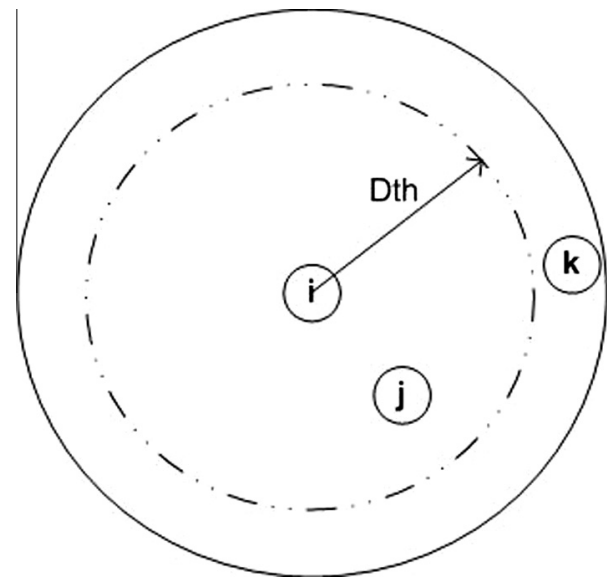

Fig. 14. Distance threshold in Discount scheme.

include the nodes that have already been covered. During the waiting time $R A D$, nodes can receive multiple broadcast packets from their neighbors. The nodes collect which neighbors have already been covered in order to make the decision of retransmitting the broadcast packet again. If all of a node's neighbors are covered during the waiting time, the node will not forward the broadcast packet. Otherwise, the retransmission probability will be:

$p=p_{i} e^{-k m}$

where $k$ is a constant to control the forwarding probability.

3.2.2.4. Energy-based schemes. GEM scheme combines counter-based, energybased and distance-based mechanisms to adjust the forwarding probability [71]. On receiving a broadcast packet a node checks its remaining energy (energy-based mechanism), if it is lower than the threshold energy (cut-off energy), it will discard the packet. Otherwise, the node will calculate the relaying probability as a function of the Euclidean distance (distance-based mechanism) between the sender and the receiver as,

$p=\frac{d}{r} p_{h}$

where $p_{h}$ is the maximum forwarding probability. If a node has an energy level higher than the cut-off energy but it does not relay the incoming packet $x$ because its coin toss landed "tails", the node will wait for a period of time $R A D$ in order to receive a copy of $x$ from one of its neighbors. If after the waiting time the node does not receive any copy of $x$ it will try to broadcast $x$ using (70) (counter-based mechanism). One important task of this scheme is how to set the cut-off energy. The cut-off energy is a threshold used by each node to determine whether it is localized inside a low energy region. When a given node is located in a low energy region, it should not rebroadcast. The authors used the average energy level of nodes covering a certain region. A region is considered a low energy region when the mean energy of all nodes that cover this region is smaller than the network's mean energy $E_{\text {avg }}$ minus its standard deviation $\sigma$. A given node $I$ can monitor its energy coefficient $E_{c_{i}}$ using the following expression:

$E_{c_{i}}=\sum_{j=1}^{j=N_{b_{I}}} \frac{E_{j}}{n_{b+1}}+\frac{E_{I}}{n_{b}+1}$

where $E$ is the node's energy level and $N_{b_{1}}$ is the set of I's neighbors. Using $E_{a v g}, \sigma$, and $E_{c_{i}}$, the cut-off energy is determined as the highest coefficient that is smaller than $E_{a v g}-\sigma$. Notice that when this coefficient does not exist, the network does not have low energy regions so the cut-off energy is zero.

\section{Evaluation of probabilistic schemes}

This section is aimed to describe the methodology used to evaluate the performance of probabilistic broadcast in wireless ad hoc networks. This methodology includes network simulators, performance metrics, and real implementations.

\subsection{Simulators}

So far, simulation results seem to be the only means to evaluate the performance of probabilistic broadcast schemes. Because of the complexity of developing real testbeds, the simulation results have served as the main benchmark for ad hoc networks wireless networks such as WSNs, MANETs, VANETs. Although it has been observed that different simulators can produce different results for flooding algorithms [100], network simulators are still the main

Table 10

Adaptive counter-based distance-based probabilistic schemes.

\begin{tabular}{|c|c|c|c|c|}
\hline Scheme & Forwarding probability & Collected data & Adjusted parameters & Application \\
\hline DDAPF [56] & \multirow{2}{*}{$p=p_{i} e^{k_{1} \frac{S_{\min }^{(m)-S_{\max }}}{\pi r^{2}}} e^{-k_{2} m}$} & $d_{r}$ and $m$ & $k, p_{h}$, and $p_{l}$ & MANET \\
\hline ACCPF [69] & & $d$ and $m$ & $p_{i}, k_{1}$, and $k_{2}$ & MANET \\
\hline \multirow[t]{2}{*}{ Discount [49] } & 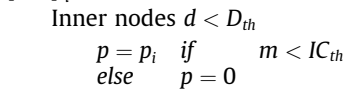 & $d$ and $m$ & $D_{t h}$, and $I C_{t h}$ & MANET \\
\hline & $\begin{array}{l}\text { Perimeter nodes } d>D_{t h} \\
\quad p=p_{i}\end{array}$ & & & \\
\hline \multirow[t]{2}{*}{ Discount RS [49] } & Inner nodes $d<D_{t h}$ & $d$ and $m$ & $D_{t h}$, and $I C_{t h}$ & MANET \\
\hline & 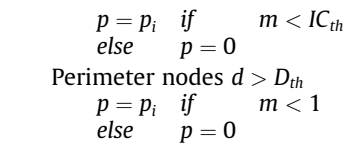 & & & \\
\hline
\end{tabular}


evaluation method for assessing broadcast schemes. The Network Simulator 2 (NS-2) [38] is so far the most used simulator for evaluating broadcast and routing algorithms in wireless ad hoc networks. However, other network simulators have also been used to evaluate broadcast schemes such as GlomoSim [124], QualNet, OPNET, and JiST/SWANS $[14,15]$. On the other hand, a few experimental results have been provided in the last few years. Experimental results are important to corroborate and validate the analytical models and the simulation results.

\subsection{Performance metrics}

The objective of this subsection is to present a detailed list of metrics widely used for the evaluation of broadcast schemes. Notice that although the following metrics have been used to evaluate probabilistic broadcast schemes, they can also be used to evaluate other types of broadcast schemes. We have divided the evaluation metrics into four groups, see Fig. 15, (1) Broadcast efficiency, (2) Discovery process, (3) Routing protocols, and (4) MAC layer. The broadcast efficiency metrics evaluate the broadcast schemes as a stand-alone dissemination technique. These metrics are especially useful for the cases when broadcast schemes are used to disseminate warning messages. We have classified the broadcast efficiency metrics into four categories, (1) Reachability, (2) Redundancy, (3) delay, and (4) energy. The reachability metrics measure the outreach of the broadcast process. The redundancy metrics determine the overhead caused by the broadcast schemes. In general, low redundancy is a basic requirement for broadcast schemes. Delay metrics calculate the time elapsed during the broadcast process. Delay needs to be small when broadcast schemes are used to disseminate warning messages. Finally, energy metrics evaluate the broadcast schemes in terms of energy consumption, which is of paramount importance in wireless ad hoc networks.

The discovery process metrics evaluate the broadcast schemes when they are used in the discovery process of routing protocols. In this case, the objective is to find a communication path between a source node and a destination node in the network. Notice that it is different from the objective of stand-alone broadcast schemes whose main objective is to reach as many nodes as possible.

The routing protocols metrics are normally used to evaluate the routing protocols in wireless ad hoc networks [62]. Since broadcast schemes are an important part of routing protocols, they impact on the general performance of routing protocols. MAC layer metrics evaluate the broadcast schemes in terms of number of collisions. As mentioned earlier, simple flooding causes the well-known broadcast storm problem [109,108] so MAC layer metrics give an idea on how the broadcast problem is alleviated when probabilistic broadcast schemes are employed.

The metrics included in Fig. 15 are defined as follow:

\subsubsection{Broadcast efficiency metrics}

4.2.1.1. Reachability. The objective of this metrics consists of evaluating the dissemination of the broadcast message throughout the network, so high values of the following metrics are always desired in a broadcast scheme.

- Reachability $(R e)$ : is defined as the ratio of nodes that received the broadcast packets to the total number of nodes in the network. When the network is not fully connected, the reachability metric is defined as the ratio of nodes that received the broadcast packets to the total number of nodes that can be reached by the source node directly or through a

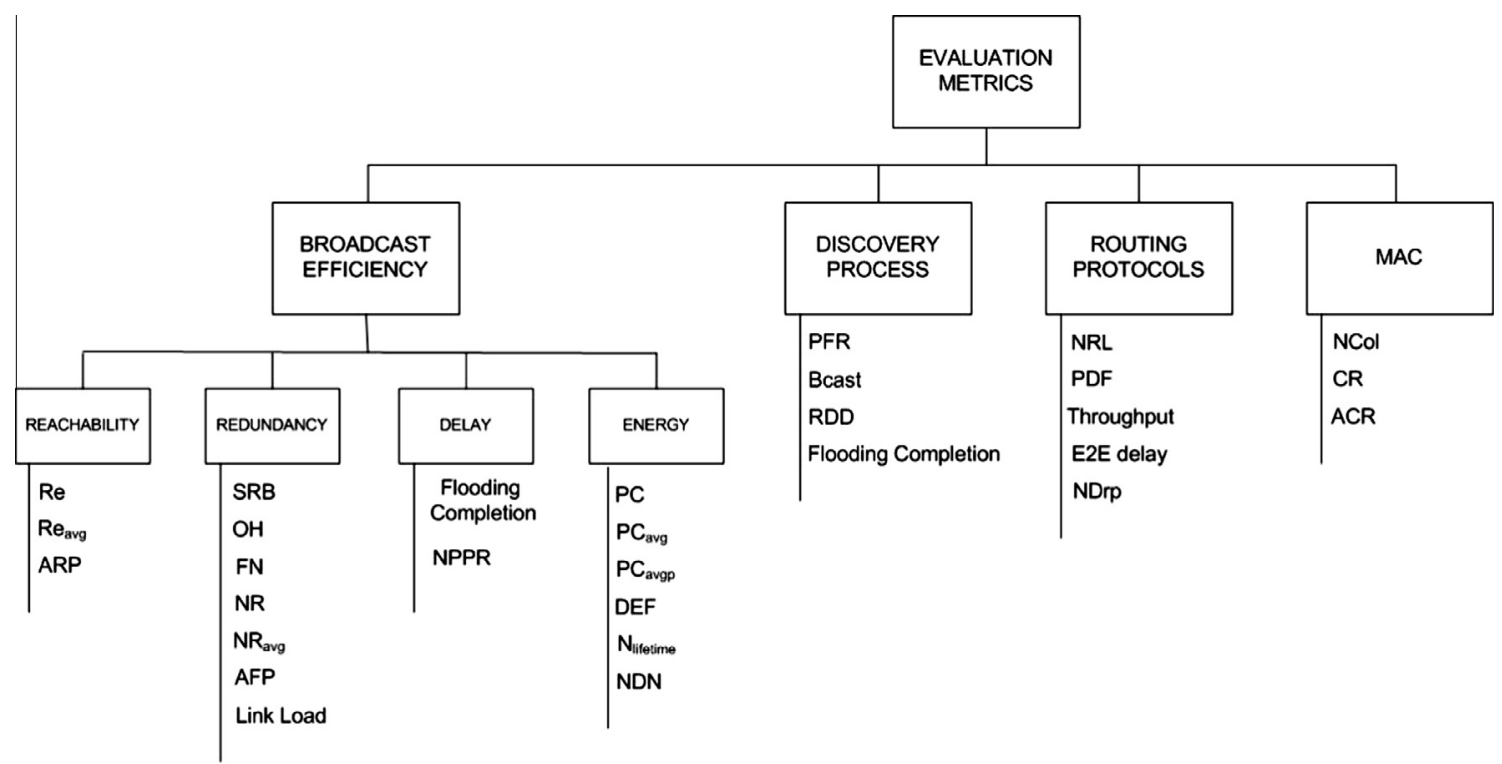

Fig. 15. Classification of the evaluation metrics. 
multi-hop path. This metric is also referred to as the fraction of nodes, Broadcast Delivery Ratio (BDR), and Broadcast Coverage Ratio (BCR) or Coverage.

- Average Reachability per broadcasting $\left(R e_{a v g}\right)$ : Average reachability of the broadcast processes executed.

- Average Reception Percentage $(A R P)$ : Reception percentage of a given node with respect to the source node, is the percentage of messages originated at the source node and received at the destination node. The ARP is the reception percentage, averaged over all nodes.

\subsubsection{Redundancy}

The following metrics evaluate broadcast schemes in terms of redundancy. In general, redundancy should be maintained as low as possible in broadcast schemes. The ideal scenario is one where nodes only receive a broadcast packet once.

- Saved ReBroadcast (SRB): Let $N_{r}$ be the number of nodes that received the broadcast message and let $N_{t}$ be the number of nodes that actually transmitted the message. The saved rebroadcast is then defined by $\frac{N_{r}-N_{t}}{N_{r}}$.

- Redundancy Overhead $(\mathrm{OH})$ : The number of duplicated packets received at each node divided by the total number of nodes in the network.

- Number of forwarding nodes $(F N)$ : The number of duplicated packets received at each node divided by the total number of nodes in the network.

- Number of retransmissions (NR): The total number of retransmissions. This metric is also referred to as number of rebroadcasts.

- Average retransmissions per broadcasting $\left(N R_{a v g}\right)$ : The average number of retransmissions per broadcasting. This is very similar to the previous term, but it also considers the number of broadcast process.

- Average Forwarding Percentage $(A F P)$ : Forwarding percentage of a given node with respect to the source node, is the percentage of messages originated at the source node and forwarded by the node. The AFP is the reception percentage, averaged over all nodes.

- Efficiency Rate (ER): This metric is defined as the forwarding rate divided by the reception rate.

- Link Load: The link load measures the amount of broadcast traffic received at each node over a unit time.

4.2.2.1. Delay. Delay metrics are related to the time spent by a broadcast message to cross the network. As a rule, low delay is always a good feature of a broadcast scheme specially when the broadcast scheme is used to disseminate emergency messages.

- Normalized Packet Penetration Rate (NPPR): This metric represents how fast the broadcast packets travel across the network.
- Flooding Completion Time or Broadcast end to end delay: The time elapsed between the first broadcast of a broadcast packet and the received route reply.

4.2.2.2. Energy. The following metrics are used to measure the power consumption of broadcast schemes. These metrics are specially relevant in WSNs where nodes are normally fed by batteries. In general, low power consumption is always desirable.

- Power Consumption $(P C)$ : The total power consumption of the nodes forming the network.

- Average Power Consumption $\left(P C_{a v g}\right)$ : The average power consumption of the broadcast processes executed.

- Average Power Consumption per Broadcasting $\left(P C_{a v g_{p}}\right)$ : The average power consumption of the broadcast processes executed.

- Directional Energy Efficiency $(D E F)$ : The ratio of the number of non duplicate packets to the number of sectors used for forwarding broadcast packets (this metric is only used in directional broadcast [104]).

- Network Lifetime $\left(N_{\text {lifetime }}\right)$ : The network lifetime is defined as the time it takes for the first node to deplete its energy. Notice that this metric sometime is defined as the time it takes for a percentage of the network to deplete its energy.

- Number of Dead Nodes (NDN): The total number of dead nodes in the network. A node is dead when its battery level has depleted.

\subsubsection{Discovery process}

The following metrics are used to evaluate broadcast schemes as part of the discovery phase of routing protocols. The objective consists of finding a communication path between a source node and a destination node or several destination nodes. The basic requirements are: finding a communication path as fast as possible, using the lowest possible resources (messages) and with a high rate of success.

- Path Found Ratio (PFR): The ratio of the number of paths found to the number of path requests. This metric is also defined as Discovery Rate and Connectivity Success Ratio.

- Route Discovery Delay $(R D D)$ : The time elapsed between the first broadcast of a request packet and the received route reply.

- Bcast: The average number of broadcast packets sent by a node per path discovered.

\subsection{Routing protocols}

The following metrics are normally used to evaluate routing protocols in ad hoc networks. Consequently, they can also be used to evaluate broadcast schemes since they are an important part in the performance of routing protocols. 
- Number of hops: The total number of hops from the source node to the destination node.

- Packet Delivery Fraction (PDF): The ratio of packets received successfully by the source node.

- End-to-End Delay (E2E) or Latency: The delay experienced by a packet from the time it is sent by the source until the time it reached the destination.

- Normalized Routing Load (NRL): The number of routing packets transmitted for every data packet sent. This metric is also referred to as routing overhead.

- Throughput: Total data successfully received by their destinations divided by the simulation time.

- Number of Dropped Packets (NDrp): The total number of dropped packets.

\subsubsection{MAC layer}

The following metrics are normally used to evaluate MAC protocols, but they can also evaluate the performance of broadcast schemes since the number of collisions will be influenced by the broadcast scheme used. As a rule, low number of collisions is a requirement for a broadcast scheme.

- Number of Collisions (NCol): The total number of collisions.

- Collision Rate $(C R)$ : The total number of broadcast packets dropped by the MAC layer as a result of collisions per unit simulation time.

- Average Collision Rate $(A C R)$ : The average number of broadcast packets dropped by the MAC layer as a result of collisions per unit simulation time.

\subsection{Comparison}

In this subsection, we present the most relevant information that we have obtained from the analysis of the result sections of the papers reviewed in this survey, considering how the comparison among different broadcast schemes have been carried out. The main findings are:

- Most of the proposed broadcast schemes in the literature are only compared to simple flooding and GOSSIP1 [41,42], see Fig. 16. Consequently, there is a lack of a complete comparison among different probabilistic broadcast schemes proposed in the literature. When other schemes are used in the evaluation they are normally proposed by the same authors so there is little comparison among schemes proposed by different authors.

- Most of the proposed broadcast schemes have been proposed for MANETs as can be observed in Fig. 17. However, it would be useful to evaluate the proposed schemes for different target scenarios since the goals of broadcast schemes different types of ad hoc networks are very similar.

- As for the performance metrics, Re, SRB, E2E are the most used metrics to evaluate broadcast schemes whenever they are proposed as standalone dissemination mechanisms. On the other hand, if the broadcast schemes are evaluated as part of the discovery phase of routing protocols, NRL and PDF are the preferred performance metrics.

- When mobility of nodes is considered, for instance in MANETs, the RWP [30] model is so far the most used mobility model. Although this mobility model has been criticized for being unrealistic [123], it is still widely used to evaluate the routing and broadcast algorithms. Moreover, the steady-state version of RWP [81] solves the issues observed in the traditional version of RWP [123]. In VANET scenarios, the mobility models simulate vehicles in a highway with different lanes. On the other hand, static scenarios are normally employed in WSNs. The use of real trace-based mobility model would be useful to evaluate the performance of probabilistic broadcast schemes in real scenarios $[78,10]$.

- As mentioned earlier, the network simulator NS-2 [38] is the de facto simulator for evaluating probabilistic broadcast schemes in wireless networks. The usage of networks simulators is illustrated in Fig. 18.

- In general, the use of hello packets is widely applied to probabilistic broadcast schemes. The period of hello packets should be adjusted carefully since it impacts on the congestion of the network [122]. In addition, when neighboring information is included in the hello packets, it increases the payload that in turns affect the time duration for which the packets occupy the wireless medium.

- AODV routing protocol [91] is the most used routing protocol to evaluate the performance of the proposed probabilistic broadcast schemes when they are implemented on a routing protocol as part of the discovery process.

- The IEEE 802.11 Distributed Coordination Function (DCF) [1] is the standard MAC layer employed in majority of the proposed broadcast schemes found in the literature. However, there are many other proposed schemes that are evaluated under an ideal MAC layer. It means that packet collisions are not taken into consideration. Although considering an ideal MAC layer can be useful for evaluating the efficiency of the proposed algorithms, a realistic MAC layer is necessary to emulate real-life scenarios. Moreover, the broadcast schemes impact on the congestion and contention of the network so a real MAC layer is necessary to model and assess the efficiency of a broadcast scheme.

\subsection{Real implementations}

Little work has been focused on the evaluation of broadcast schemes in general and probabilistic broadcast schemes in particular. Flooding is studied in detail by Ganesan et al. [39] in a grid scenario (a grid with 150 nodes), and the results showed that even in dense scenarios the reachability is not $100 \%$. In $[20,22]$ the Testbed (DES-Testbed) [23] is used to evaluate several probabilistic schemes. In $[20,22]$, the DES-Testbed is composed of 59 mesh wireless routers located in two adjacent buildings. In [22] 


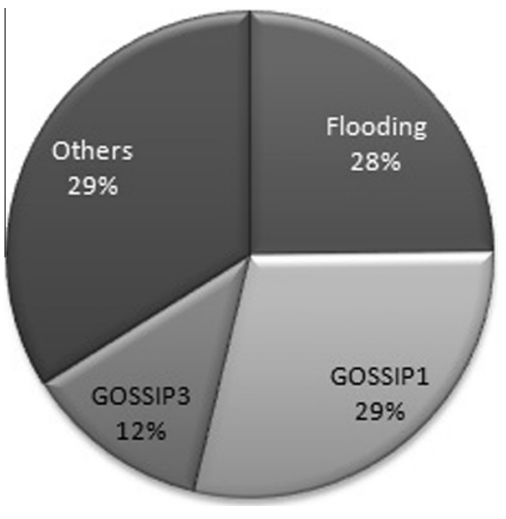

Fig. 16. Comparison schemes.

where the probabilistic schemes used are GOSSIP1, two thresholds scheme (GOSSIP2), GPSSIP3, and GOSSIP5, the main findings are:

- The bimodal behavior is observed in GOSSIP1 for a forwarding probability about 0.7 . These results corroborate the results included in [41,42]. However, the results in [22] indicate that such behavior depends on the position of the source node.

- The authors observed that even when a forwarding probability equal to 1 is used the maximum reachability of $100 \%$ is not achieved.

- The results obtained by GOSSIP2 showed that this scheme has problems in nodes with a low degree so it can cause network partitioning.

- GOSSIP3 is the probabilistic scheme that achieved the best results in terms of reachability.

- GOSSIP5, it exhibited a behavior strongly dependent on the source node's position so the authors stated that this scheme needed further research.

On the other hand, in [20] when the probabilistic schemes (adapted to the target scenario conditions) used are GOSSIP3 [42,41], AGAR [105], the dynamic probabilistic scheme based on self-pruning [3], PCBR [76], and APF $[44,45]$, the main findings are:
- The experimental results showed that GOSSIP3 achieved the best results. GOSSIP3 exhibits independence of the forwarding probability. Moreover, it does not need to exchange hello packets periodically.

- The rest of the probabilistic schemes showed some problems to adapt the adjusting parameters to the deployed scenario. The authors observed that the adjusting parameter should be chosen carefully in order to achieve satisfactory results.

- The authors noticed the importance of the position of the source node.

- According to the results included in [20], none of the probabilistic schemes achieved reachability higher than $80 \%$ and the saved rebroadcast compared to flooding is up to $30 \%$.

- The authors also corroborated $h=1$ as a suitable value for the counter-based scheme's threshold, which is previously demonstrated by simulations [42].

- Finally, the authors pointed out that the most important issue in probabilistic schemes is to identify which nodes are vital for the connectivity of the network.

In a later work Blywis et al. [21], analyzed the limits of some probabilistic schemes experimentally. They used again DES-Testbed, but in this case 105 nodes are used. In particular, they focused their work on GOSSIP1 and GOSSIP3. They studied how some traffic characteristics, such as congestion and payload impact on the performance of GOSSIP1 and GOSSIP3 schemes. The main finding of this study are:

- In GOSSIP1 reachability increases with the forwarding probability and decreases with the congestion (number of sources) and the payload.

- The performance of GOSSIP3 worsens with probability, congestion, and payload. If the forwarding probability is increased in GOSSIP3, the network would behave similar to the situation when flooding is used.

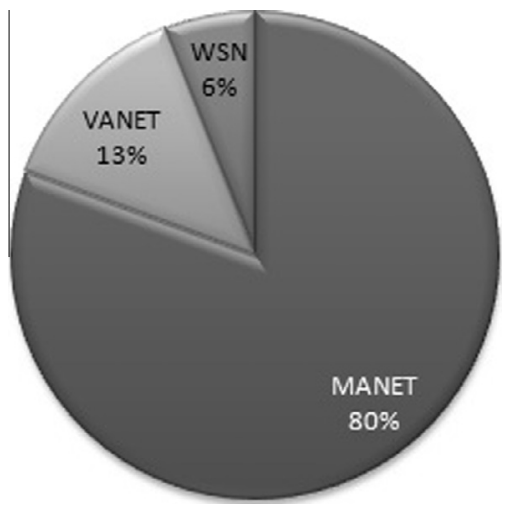

Fig. 17. Target network.

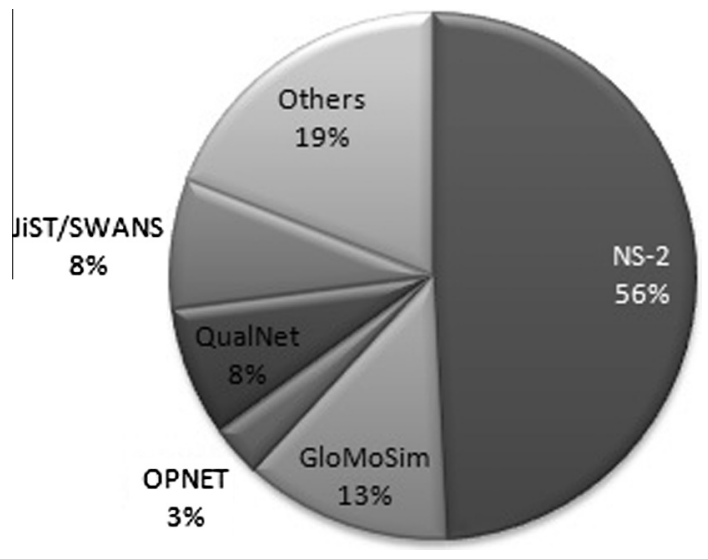

Fig. 18. Network simulators. 
- The authors indicated that the suitable value of the forwarding probability for GOSSIP3 is within the interval $[0.2,0.3]$.

In recent works [24,25], the authors corroborated that real wireless ad hoc networks do not percolate due to collisions and noisy links, which is previously demonstrated by simulations in [102]. The authors proposed a suppressed bond-site model [82] for modeling the effects of collisions and noisy links in the percolation model.

Other studies that are not focused on probabilistic schemes also use probabilistic schemes for comparison purposes. In [68] the schemes adaptation hop by hop [128] and ECS [73] are compared to other deterministic broadcasting schemes.

\section{Lessons learned and open challenges}

\subsection{Lessons learned}

In this subsection we indicate the lessons learned in this survey. The main lessons are:

- Probabilistic broadcasting in ad hoc networks (as a dissemination technique and as part of routing protocols) is an important active research area in MANETs, VANETs, and WSNs.

- The optimal probability is not universal and it depends on many topological parameters such as density, mobility, and scenario.

- According to a number of approaches presented in this survey, density metrics are the most used to adjust the forwarding probability in adaptive schemes. In non counter-based schemes, the number of neighbors is the most used density metric. On the other hand, in counter-based schemes, the key metric is the number of duplicated packets received. Although global metrics are used, they should be avoided since global information is difficult to be collected in ad hoc networks.

- Many probabilistic schemes are based on thresholds like density thresholds. However, the optimal value of such thresholds is difficult to obtain in ad hoc networks since these may depend on numerous parameters.

- Many distance-based schemes use the node's transmission range in the expressions to calculate the forwarding probability. However, this parameter can be very different from the nominal value defined by the manufacturer. Several external factors can impact on the real value of a node's transmission range such as external noise and interferences.

- With regard to the evaluation of probabilistic broadcast schemes, most proposed schemes are evaluated under MANETs scenarios with 802.11 MAC layer using the random waypoint mobility model in NS-2 simulator. When a routing protocol is used, AODV is normally the chosen routing protocol. As for the evaluation metrics, Re and SRB are the most used evaluation metric when probabilistic broadcast is used as a stand-alone dissemination technique. On the other hand, PDF, NRL and E2E delay are the most used when probabilistic broadcast is used as part of the discovery phase of routing protocols.

- There are differences between the simulation results obtained and the real experiments carried out. The main problem with some schemes is related to how the defined thresholds are configured in the real experiments.

\subsection{Open challenges}

In this subsection we indicate the open challenges that have not been covered yet or little work has been focused on them. In addition, we comment briefly how these open challenges can be addressed. The main challenges in probabilistic broadcast schemes are:

- More analytical models that predict the behavior of probabilistic broadcast schemes are needed. Although some analytical models have been presented for broadcasting schemes in wireless ad hoc networks, such as flooding [110,113], fixed probabilistic scheme $[110,113]$, distance-based $[126,113,125]$, counter-based $[126,113,125]$, location-based [113], further work is needed to model the different types of probabilistic schemes presented in this survey. Only a few papers include analytical models for the proposed schemes such as $[70,37]$. Most of the proposed schemes are based on heuristics. In [27] a framework is proposed to evaluate some probabilistic broadcast schemes (IF and NPPB schemes), however, the authors stated that it can be easily extended to evaluate other probabilistic and deterministic broadcast schemes.

- The definition of standard evaluation scenarios and their features such as density, path length (number of hops), congestion, and mobility models. Throughout the papers reviewed in this survey, the authors have used different values of density, and mobility models need to be standardized. Consequently, it is difficult to compare the simulation results presented in different papers. Standard scenarios are needed for a fair comparison of the probabilistic schemes. Kurkowski et al. [59,58], presented some works focused on constructing standard scenarios for MANETs. With regard to mobility conditions, most schemes have been evaluated using the random Waypoint mobility model. However, there are many other synthetic mobility models that can be used to evaluate probabilistic broadcast schemes [30,78]. There exist mobility generators, such as BonnMotion [9] and VanetMobiSim [47], that can be used to evaluate the probabilistic schemes under different mobility models. Moreover, we think that the use of real-life trace-based mobility $[10,78]$ models would be useful to evaluate the performance of probabilistic schemes in real-life scenarios. The 
Community Resource for Archiving Wireless Data at Dartmouth (CRAWDAD) [120] is an open project aimed to archive data collected from real-life experimentation. This data is freely available and can be used to evaluate broadcast schemes in real scenarios.

- As for the evaluation metrics, it would be useful to define a standard metric for evaluation of probabilistic schemes. In [87] the authors suggested a standard metric to evaluate broadcasting schemes in VANETs. This metric, namely Dissemination Efficiency (DE), combines redundancy, delay, reachability, and success rate. Although it is proposed for VANET scenarios, it can be used for other types of ad hoc networks.

- There is lack of a complete comparison of probabilistic schemes. According to the comparison presented in Section 4.2.1, most probabilistic schemes have only been compared to simple flooding and fixed probabilistic schemes. We hope that this survey will help to improve the understanding of probabilistic schemes and encourage the research community to address the areas where there is lack of effort. There are some examples of good comparisons among broadcast schemes such as [31,34].

- Regarding adaptive counter-based schemes, there is no a clear evaluation of the role played by RAD. There is not evaluation on the optimal value of RAD in counter-based schemes.

- In all the reviewed schemes, nodes cooperate in the broadcast process, however, it may be interesting to evaluate the broadcast schemes considering selfish nodes. That is, nodes that do not want to cooperate in the broadcast process and also malicious nodes.

- More experimental results are needed to validate the simulation results. The experimental results should corroborate the simulation results achieved. According to the experimental results presented in [20,22], it is obvious that the adjusting parameters of probabilistic scheme must be carefully selected.

- Although many probabilistic schemes have been proposed, more adaptive probabilistic schemes are needed. Many proposed schemes use global parameters like global density values. It is unfeasible that nodes could collect such global information in real life scenarios. The forwarding probability should only be adapted using local parameters of nodes like local density.

\section{Summary}

In this paper, we have highlighted the importance of broadcasting techniques in the performance of wireless ad hoc networks, showing that probabilistic broadcast methods exhibit suitable performance in mobile conditions, emergency situations, and in scenarios with limited resources. Then, we have proposed a classification for probabilistic broadcast schemes according to the reviewed literature, Figs. 1 and 2. Using the proposed classification, we have presented a comprehensive review of the probabilistic broadcast found in the literature that fall into the proposed classification. The review points out the main features and shortcomings of each specific scheme. These details can be found in the set of tables included in Section 3 (Tables 2-10). In addition, we analyzed the methodology followed by researchers to evaluate the probabilistic broadcast techniques, considering factors like networks simulators, performance metrics, and real implementations. According to the reviewed literature, simulation is still the main method for evaluating broadcast schemes in ad hoc networks, only some works presented experimental results that indicate the existence of some differences from the simulation results with respect to the experimental results. Regarding the reviewed literature, it is evident that there is lack of a complete comparison of probabilistic broadcast schemes. According to the information included in the comparison section, most probabilistic schemes are only compared to GOSSIP1 and flooding schemes. We hope that this survey helps to encourage the research community to address this issue. Finally, some guidelines are given on the open challenges that have not been addressed or where more research is needed.

\section{Acknowledgments}

This work was supported in part by the University of Seville under the Ph.D. grant PIF (Personal Investigador en Formación) of Daniel Gutiérrez Reina.

\section{References}

[1] IEEE Standard for Information Technology - Telecommunications and Information Exchange between Systems - Local and Metropolitan Area Networks - Specific Requirements - Part 11: Wireless Lan Medium Access Control (mac) and Physical Layer (phy) Specifications, June 2007.

[2] W. Abdou, A. Henriet, C. Bloch, D. Dhouutaut, D. Charlet, F. Spies, Using a evolutionary algorithm to optimize the broadcasting methods in mobile ad hoc networks, J. Network Comput. Appl. 34 (2011) 1794-1804.

[3] J.-D. Abdulai, M. Ould-Khaoua, L.M. Mackenzie, Adjusted probabilistic route discovery in mobile ad hoc networks, Comput. Electr. Eng. 35 (2009) 168-182.

[4] J.D. Abdulai, M. Ould-Khaoua, L.M. Mackenzie, A. Mohammed, Neighbour coverage: A dynamic probabilistic route discovery for mobile ad hoc networks, in: International Symposium on Performance Evaluation of Computer and Telecommunication Systems (SPECTS 2008), 2008, pp. 165-172.

[5] M. Abolhasan, T. Wysocki, E. Dutkiewicz, A review of routing protocols for mobile ad hoc networks, Ad Hoc Netw. 2 (2004) 1-22.

[6] K. Akkaya, M. Younis, A survey on routing protocols for wireless sensor networks, Ad Hoc Netw. 3 (2005) 325-349.

[7] I.F. Akyldiz, W. Su, Y. Sankarasubramaniam, E. Cayirci, Wireless sensor networks: a survey, Comput. Netw. 38 (2002) 393-422.

[8] H. Alshaer, E. Horlait, An optimized adaptive broadcast scheme for inter-vehicle communication, in: IEEE 61st Vehicular Technology Conference (VTC 2005-Spring), May 2005, pp. 2840-2844.

[9] N. Aschenbruck, R. Ernst, E. Gerhards-Padilla, M. Schwamborn, Bonnmotion-a mobility scenario generation and analysis tool, in: Proceedings of the 3rd International ICST Conference on Simulation Tools and Techniques (SIMUTools '10), 2010.

[10] N. Aschenbruck, A. Munjal, T. Camp, Trace-based mobility modeling for multi-hop wireless networks, Comput. Commun. 34 (2011) 704-714.

[11] B. Bako, I. Rikanovic, F. Kargl, E. Schoch, Adaptive topology based gossiping in vanets using position information, in: Proceedings of the 3rd international conference on Mobile ad-hoc and sensor networks (MSN'07), 2007, pp. 66-78.

[12] B. Bako, E. Schoch, F. Kargl, M. Weber, Optimized position based gossiping in vanets, in: IEEE 68th Vehicular Technology Conference (VTC 2008-Fall), 2008, pp. 1-5.

[13] M. Bani-Yassein, M. Ould-Khaoua, M. Mackenzie, S. Papanastasiou, Performance analysis of adjusted probabilistic broadcasting in 
mobile ad hoc networks, Int. J. Wireless Inf. Networks 13 (2) (2006) 127-139.

[14] R. Barr, JiST - Java in Simulation Time User Guide, 2003. <http:// jist.ece.cornell.edu/docs.html>.

[15] R. Barr, SWANS - Scalable Wireless Ad hoc Network Simulator User Guide, 2004. <http://jist.ece.cornell.edu/docs.html>.

[16] R. Beraldi, A directional gossip protocol for path discovery in manets, in: 26th IEEE International Conference on Distributed Computing Systems Workshops (ICDCS 2006), 2006.

[17] R. Beraldi, The polarized gossip protocol for path discovery in manets, Ad Hoc Netw. 6 (2006) 79-91.

[18] R. Beraldi, R. Baldoni, Unicast routing techniques for mobile ad hoc networks, in: The Handbook of Ad Hoc Wireless Networks, The Electrical Engineering Handbook Series, 2003.

[19] R. Beraldi, L. Querzoni, R. Baldoni, A hint-based probabilistic protocol for unicast communication in manets, Ad Hoc Netw. 4 (2006) 547-566.

[20] B. Blywis, M. Günes, S. Hofmann, F. Juraschek, A study of adaptive gossip routing in wireless mesh networks, Lect. Notes Inst. Comput. Sci., Social Inf. Telecommun. Eng. 49 (2010) 98-113.

[21] B. Blywis, M. Günes, F. Juraschek, O. Hahm, Challenges and limits of flooding and gossip routing based route discovery schemes, in: The 36th IEEE Conference on Local Computer Networks, October 2011 pp. 283-286.

[22] B. Blywis, M. Günes, F. Juraschek, S. Hofmann, Gossip routing in wireless mesh networks, in: IEEE 21st International Symposium on Personal Indoor and Mobile Radio Communications (PIMRC 2010), 2010, pp. 1572-1577.

[23] B. Blywis, M. Günes, F. Juraschek, P. Schmidt, P. Kumar, Des-sert: a framework for structured routing protocol implementation, in: IFIP Wireless Days, 2009

[24] B. Blywis, P. Reinecke, M. Günes, K. Wolter, Gossip routing, percolation, and restart in wireless multi-hop networks, Tech. Rep. TR-B-11-08, Institute of Computer Science, Freie Universitat Berlin, Germany, October 2011.

[25] B. Blywis, P. Reinecke, M. Günes, K. Wolter, Gossip routing percolation, and restart in wireless multi-hop networks, in: IEEE Wireless Communication and Networking Conference, 2012, pp. 3019-3023.

[26] A. Boukerche, B. Turgut, N. Aydin, M.Z. Ahmad, L. Boloni, D. Turgut, Routing protocols in ad hoc networks: a survey, Comput. Netw. 55 (2011) 3032-3080.

[27] S. Busanelli, G. Ferrari, R. Gruppini, Recursive analytical performance evaluation of broadcast protocols with silencing: application to vanets, EURASIP J. Wireless Commun. Networking 10 (2012) 1-21.

[28] S. Busanelli, G. Ferrari, S. Panichpapiboon, Efficient broadcasting in ieee 802.11 networks through irresponsible forwarding, in: IEEE Global Telecommunications Conference (GLOBECOM 2009), December 2009, pp. 1-6.

[29] S. Busanelli, G. Ferrari, S. Panichpapiboon, Cluster-based irresponsible forwarding, in: The internet of things: 20th Tyrrhenian Workshop on Digital Communications, 2010, pp. 59-68.

[30] T. Camp, J. Boleng, V. Davies, A survey of mobility models for ad hoc networks research, Wireless Commun. Mobile Comput.; Special Issue Mobile Ad Hoc Networks; Res. Trends Appl. 2 (5) (2002) 483502.

[31] T. Camp, B. Williams, Comparison of broadcasting techniques for mobile ad hoc networks, in: Proceeding of the ACM International Symposium on Mobile Ad Hoc Networking and Computing, 2002, pp. 194-205.

[32] J. Cartigny, D. Simplot, Border node retransmission based probabilistic broadcast protocols in ad-hoc networks, in: Proceedings of the 36th Annual Hawaii International Conference on System Sciences (HICSS '03), 2003.

[33] J. Cartigny, D. Simplot, Border node retransmission based probabilistic broadcast protocols in ad-hoc networks, Telecommun. Syst. 22 (1-4) (2003) 189-204.

[34] F. Dai, J. Wu, Performance analysis of broadcast protocols in ad hoc networks based on self-pruning, IEEE Trans. Parallel Distrib. Syst. 15 (11) (2004) 1027-1039.

[35] D. Dembla, Y. Chaba, Recent Trends in Network Security and Applications, vol. 89, Springer, 2010. pp. 518-527, (Chapter: Modeling and Performance Analysis of Efficient and Dynamic Probabilistic Broadcasting Algorithm in MANETs Routing Protocols).

[36] V. Drabkin, R. Friedman, G. Kliot, M. Segal, Rapid: reliable probabilistic dissemination in wireless ad hoc networks, in: 26 th
IEEE International Symposium on Reliable Distributed Systems, 2007, pp. 13-22.

[37] V. Drabkin, R. Friedman, G. Kliot, M. Segal, On reliable dissemination in wireless ad hoc networks, IEEE Trans. Dependable Sec. Comput. 8 (6) (2011) 866-882.

[38] K. Fall, K. Varadhan, In the ns manual (formerly ns notes and documentation). documentation.html>.

[39] D. Ganesan, B. Krishnamachari, A. Woo, D. Culler, D. Estin, S. Wicker, Complex Behaviour at Scale: An Experimental Study of Low-Power Wireless Sensor Networks. Tech. Rep. UCLACSD-TR 020013, Computer Science Departament, UCLA, 2002.

[40] A.K. Gogada, B. Santosh, R. Garimella, A simple tsunami warning system based on wireless sensor networks, in: Proceedings of the 4th International Conference on Distributed Computing and Internet Technology (ICDCIT'07), 2007.

[41] Z.J. Haas, J.Y. Halpern, L. Li, Gossip-based ad hoc routing, in: IEEE InfoCom Proceedings, vol. 3, 2002, pp. 1707-1716.

[42] Z.J. Haas, J.Y. Halpern, L. Li, Gossip-based ad hoc routing, IEEE/ACM Trans. Networking 14 (3) (2006) 479-491.

[43] Z.J. Haas, M.R. Pearlman, The performance of query control schemes for the zone routing protocol, IEEE/ACM Trans. Networking 9 (4) (2001) 427-438

[44] A.M. Hanashi, I. Awan, M. Woodward, Performance evaluation based on simulation of improving dynamic probabilistic flooding in manets, in: International Conference on Advanced Information Networking and Applications Workshops, 2009, pp. 458-463.

[45] A.M. Hanashi, A. Siddique, I. Awan, M. Woodward, Performance evaluation of dynamic probabilistic broadcasting for flooding in mobile ad hoc networks, Simul. Model. Pract. Theory 17 (2009) 364-375

[46] W. Hardle, L. Simar, Applied multivariate statistical analysis, Method Data Technol. (2003).

[47] J. Harri, F. Filali, C. Bonnet, Vanetmobisim: Generating realistic mobility patterns for vanets, in: Proceedings of the 3rd International Workshop on Vehicular Ad Hoc Networks (VANET '06), 2006, pp. 96-97.

[48] J. Hoebeke, I. Moerman, B. Dhoedt, P. Demeester, An overview of mobile ad hoc networks: applications and chanllenges, J. Commun Networks 3 (2004) 60-66.

[49] C.-K. Hsu, C. Chen, H.-K. Wang, Discount: a hybrid probabilitybased broadcast scheme for wireless ad hoc networks, in: IEEE 62nd Vehicular Technology Conference (VTC-2005-Fall), 2005, pp. 2706-2710.

[50] T. Huang, S. Chen, L. Tang, Energy-aware routing for mobile ad hoc networks, in: IEEE International Conference on High Performance Computing and Communications, 2011, pp. 955-959.

[51] A. Jamal-deen, M. Aminu, N. Kaku, O. Ekanah, Route discovery in wireless mobile ad hoc networks with adjusted probabilistic flooding, in: 2nd International Conference on Adaptive Science \& Technology, 2009, pp. 99-109.

[52] M. Jelasity, S. Voulgrais, R. Guerraoui, A.M. Kermarrec, M. van Steen, Gossip-based peer sampling, ACM Trans. Comput. Syst. 25 (3) (2007) 1-36.

[53] H. Jeong, J. Kim, Y. Yoo, Adaptive broadcasting method using neighbor type information in wireless sensor networks, Sensors 11 (2011) 5952-5967.

[54] N. Karthikeyan, V. Palanisamy, K. Duraiswamy, Optimum density based model for probabilistic flooding protocol in mobile ad hoc network, Eur. J. Sci. Res. 39 (4) (2010) 577-588.

[55] A. Keshavarz-Haddad, V. Ribeiro, R. Riedi, Color-based broadcasting for ad hoc networks, in: 4th International Symposium on Modeling and Optimization in Mobile, Ad Hoc, and Wireless Networks, 2006.

[56] I.A. Khan, A. Javaid, H.L. Qian, Distance-based dynamically adjusted probabilistic forwarding for wireless mobile ad hoc networks, in: 5 th IFIP International Conference on Wireless and Optical Communications Networks (WOCN '08), June 2008.

[57] A. Khelil, P.J. Marron, C. Becker, K. Rothermel, Hypergossiping: a generalized broadcast strategy for mobile ad hoc networks, Ad Hoc Netw. 5 (2007) 531-546.

[58] S. Kurkowski, W. Navidi, T. Camp, Two standards for rigorous manet routing protocol evaluation, in: Proceedings of the 3rd IEEE International Conference on Mobile Ad Hoc and Sensor Systems, 2006, pp. 256-266.

[59] S. Kurkowski, W. Navidi, T. Camp, Constructing manet simulation scenarios that meet standards, in: IEEE Internatonal Conference on Mobile Adhoc and Sensor Systems (MASS 2007), October 2007, pp. $1-9$. 
[60] P. Kyasanur, R.R. Choudhury, I. Gupta, Smart gossip: An adaptive gossip-based broadcasting service for sensor networks, in: Mobile Ad Hoc and Sensor Systems (MASS '06), 2006a, pp. 91-100.

[61] P. Kyasanur, R.R. Choudhury, I. Gupta, Smart Gossip: Infusing Adaptivity into Gossiping Protocols for Sensor Networks. Tech. rep., University of Illinois at Urbana-Champaign, 2006.

[62] L. Layuan, L. Chunlin, Y. Peiyan, Performance evaluation and simulations of routing protocols in ad hoc networks, Comput. Commun. 30 (2007) 1890-1898.

[63] A. Lee, I. Ra, Adaptive-gossiping for an energy-aware routing protocol in wireless sensor networks, in: Proceedings of the 6th International Wireless Communications and Mobile Computing Conference (IWCMC 2010), 2010, pp. 1131-1135.

[64] X. Li, K. Moaveninejad, O. Frieder, Regional gossip routing for wireless ad hoc networks, Mobile Networks Appl. 10 (2005) 61-77.

[65] O. Liang, Y.A. Sekercioglu, N. Mani, A survey of multipoint relay based broadcast schemes in wireless ad hoc networks, IEEE Commun. Sur. Tutorials 8 (4) (2006) 30-46.

[66] D. Liarokapis, A. Shahrabi, A probability-based adaptive scheme for broadcasting in manets, in: Proceedings of the 6th International Conference on Mobile Technology, Applications and Systems, 2009, pp. 1-6.

[67] D. Liarokapis, A. Shahrabi, Fuzzy-based probabilistic broadcasting in mobile ad hoc networks, in: Wireless Days (WD), 2011 IFIP, 2011, pp. $1-6$.

[68] Y.-D. Lin, S.-L. Chang, S.-H. Tao, J.-H. Yeh, Realizing and benchmarking broadcast algorithms in wireless mesh networks, Comput. Commun. 34 (2011) 1169-1181.

[69] H. Ling, D. Mossãl', T. Znati, Coverage-based probabilistic forwarding in ad hoc routing, in: Proceedings of 14th International Conference on Computer Communications and Networks (ICCCN 2005), October 2005, pp. 13-18.

[70] I.S. Lysiuk, Z.J. Haas, Controlled gossiping in ad hoc networks, in: IEEE Wireless Communications and Networking Conference (WCNC 2010), 2010, pp. 1-6.

[71] M. Machado, R.A.F. Mini, A.A.F. Loureiro, D.L. Guidoni, P.O. Melo, Gossiping using the energy map in wireless sensor networks, in: Proceedings of the 10th ACM Symposium on Modeling, Analysis, and Simulation of Wireless and Mobile Systems, 2007, pp. 368-372.

[72] S. Merkel, S. Mostaghim, H. Schmeck, Hop count based distance estimation in mobile ad hoc networks âĂş challenges and consequences, Ad Hoc Netw. 15 (2014) 39-52.

[73] A. Mohammed, M. Ould-Khaoua, L.M. Mackenzie, J. Abdulai, Improving the performance of counter-based broadcast scheme for mobile ad hoc networks, in: IEEE International Conference on Signal Processing and Communications (ICSPC 2007), 2007, pp. 1403-1406.

[74] A. Mohammed, M. Ould-Khaoua, L.M. Mackenzie, J. Abdulai, An adjusted counter-based broadcast scheme for mobile ad hoc networks, in: Tenth International Conference on Computer Modeling and Simulation (UKSIM 2008), 2008, pp. 441-446.

[75] A. Mohammed, M. Ould-Khaoua, L.M. Mackenzie, J.-D. Abdulai, Dynamic probabilistic counter-based broadcasting in mobile ad hoc networks, in: 2nd International Conference on Adaptive Science \& Technology (ICAST 2009), 2009, pp. 120-127.

[76] A. Mohammed, M. Ould-Khaoua, L.M. Mackenzie, C. Perkins, J.-D. Abdulai, Probabilistic counter-based route discovery for mobile ad hoc networks, in: Proceedings of the 2009 International Conference on Wireless Communications and Mobile Computing (IWCMC 2009), 2009, pp. 1335-1339.

[77] A. Mostafa, A.M. Vegni, D.P. Agrawal, A probabilistic routing by using multi-hop retransmission forecast with packet collisionaware constraints in vehicular networks, Ad Hoc Netw. 14 (2014) 118-129.

[78] M. Musolesi, C. Mascolo, Mobility models for systems evaluation, Middleware Network Eccentric Mobile Appl. (2009).

[79] Y. Mylonas, M. Lestas, A. Pitsillides, Speed adaptive probabilistic flooding in cooperative emergency warning, in: Proceedings of the 4th Annual International Conference on Wireless Internet (WICON'08), 2008.

[80] M. Naserian, K. Tepe, Game theoretic approach in routing protocol for wireless ad hoc networks, Ad Hoc Netw. 7 (2009) 569-578.

[81] W. Navidi, T. Camp, Stationary distributions for the random waypoint mobility model, IEEE Trans. Mobile Comput. 3 (1) (2004) 99-108.

[82] L. Nduwayo, R. Lindebaum, N. Chetty, Suppressed bond-site percolation, Comput. Phys. Commun. 180 (4) (2008) 503-508.

[83] D. Nitnaware, P. Karma, A. Verma, Performance analysis of energy constraint gossip based routing protocol under stochastic traffic, in:
IEEE Second International Conference on Emerging Trends in Engineering and Technology (ICETET-09), 2009, pp. 1110-1114.

[84] D. Nitnaware, A. Verma, Energy based gossip routing algorithm for manets, in: IEEE International Conference on Recent Trends in Information, Telecommunication and Computing, 2010, pp. 23-27.

[85] F. Nourazar, M. Sabaei, Dapf: an efficient flooding algorithm for mobile ad-hoc networks, in: International Conference on Signal Processing Systems, 2009, pp. 594- 598.

[86] M.J. Osborne, An Introduction to game theory, Publicly-Available Solutions (2004).

[87] S. Panichpapiboon, Irresponsible forwarding under general intervehicle spacing distributions, in: 8th International Conference on Electrical Engineering/Electronics, Computer, Telecommunications and Information Technology (ECTI-CON 2011), May 2011, pp. 357-360.

[88] S. Panichpapiboon, L. Cheng, Irresponsible forwarding under real inter-vehicle spacing distribution, IEEE Trans. Veh. Technol. 62 (2013) 2264-2272.

[89] S. Panichpapiboon, G. Ferrari, Irresponsible forwarding, in: 8th International Conference on ITS Telecommunications (ITST 2008), October 2008, pp. $311-316$.

[90] N. Patwari, J.N. Ash, S. Kyperountas, A.O.H. III, R.L. Moses, N.S. Correal, Locating the noes (cooperative localization in wireless sensor networks), IEEE Signal Process. Mag. 2 (4) (2005) 54-69.

[91] C.E. Perkins, E.M. Royer, Ad hoc on-demand distance vector routing, in Procedding of IEEE Workshop on Mobile Computing System and Applications (WMCSA), 1999, pp. 1-11.

[92] C. Raffelsberger, H. Hellwagner, Evaluation of manet routing protocols in a realistic emergency response scenario, in: 10th International Workshop on Intelligent Solutions in Embedded Systems, 2012, pp. 88-92.

[93] D.G. Reina, P. Johnson, S. Toral, F. Barrero, Optimization of network lifetime through energy-efficient broadcast scheme using dynamic random walk, in: 15th International Power Electronics and Motion Control Conference (EPE-PEMC 2012 ECCE), 2012.

[94] D.G. Reina, J.M. Leon-Coca, S.L. Toral, E. Asimakopoulou, F. Barrero, P. Norrington, N. Bessis, Multi-objective performance optimization of a probabilistic similarity/dissimilarity-based broadcasting scheme for mobile ad hoc networks in disaster response scenarios, Soft. Comput. (2014).

[95] D.G. Reina, S.L. Toral, F. Barrero, N. Bessis, E. Asimakopoulou, Evaluation of ad hoc networks in disaster scenarios, in: Third International Conference on Intelligent Networking and Collaborative Systems (INCoS, 2011), November 2011, pp. 759-764.

[96] D.G. Reina, S.L. Toral, F. Barrero, N. Bessis, E. Asimakopoulou, Modelling and assessing ad hoc networks in disaster scenarios, J. Intell. Ambient Humanized Comput. (2012).

[97] D.G. Reina, S.L. Toral, P. Johnson, F. Barrero, Hybrid flooding scheme for mobile ad hoc networks, IEEE Commun. Lett. 17 (2013) 592595.

[98] D.G. Reina, S.L. Toral, P. Johnson, F. Barrero, Improving discovery phase of reactive ad hoc routing protocols using jaccard distance, J. Supercomput. 67 (2014) 131-152.

[99] J.-P. Ryu, M.-S. Kim, S.-H. Hwang, K.-J. Han, An adaptive probabilistic broadcast scheme for ad hoc networks, Lect. Notes Comput. Sci. 3079 (2004) 646-654.

[100] Y. Sasson, D. Cavin, A. Schiper, On the accuracy of manet simulators, in: Principles of Mobile Computing (POMC 2002), 2002, pp. 38-43.

[101] Y. Sasson, D. Cavin, A. Schiper, Probabilistic Broadcast for Flooding in Wireless Mobile Ad Hoc Netoworks, Tech. Rep. IC/2002/54, Swiss Federal Institute of Technology (EPFL), 2002.

[102] Y. Sasson, D. Cavin, A. Schiper, Probabilistic broadcast for flooding in wireless mobile ad hoc networks, in: IEEE Wireless Communications and Networking (WCNC 2003), vol. 2, March 2003, pp. 1124- 1130.

[103] D.J. Scott, A. Yasinac, Dynamic probabilistic retransmission in ad hoc networks, in: Proceeding of the International Conference on Wireless Networks (ICWN04), 2004.

[104] C.-C. Shen, Z-Huang, C. Jaikaeo, Directional broadcast for mobile ad hoc networks with percolation theory, IEEE Trans. Mobile Comput. 5 (4) (2006) 317-332.

[105] Z. Shi, H. Shen, Adaptive gossip-based routing algorithm, in: IEEE International Conference on Performance, Computing, and Communications, February 2004, pp. 323-324.

[106] S. Tilak, A.L. Murphy, W.B. Heinzelman, Non-uniform information dissemination for sensor networks, in: Proceedings of the 11th International Conference on Network Protocols, 2003.

[107] Y. Toor, P. Mulethaler, A. Laouiti, A.D.L. Fortelle, Vehicle ad hoc networks: applications and related technical issues, IEEE Commun. Surveys \& Tutorials 10 (2008) 74-88. 
[108] Y. Tseng, S. Ni, E. Shih, Adaptive approaches to relieving broadcast storm in a wireless moltihop mobile ad hoc networks, IEEE Trans. Comput. 52 (5) (2003) 545-557.

[109] Y.C. Tseng, S.Y. Ni, Y.S. Chen, J.P. Sheu, The broadcast storm problem in a mobile ad hoc network, Wireless Netw. 8 (2002) 153-167.

[110] K. Viswanath, K. Obraczka, Modeling the performance of flooding in wireless multi-hop ad hoc networks, Comput. Commun. 29 (2006) 949-956.

[111] Q. Wang, H. Shi, Q. Qi, A dynamic probabilistic broadcasting scheme based on cross-layer design for manets, Int. J. Modern Education Comput. Sci. 1 (2010) 40-47.

[112] A. Wegener, H. Hellbruck, S. Fischer, C. Schmidt, S. Fekete, Autocast: an adaptive data dissemination protocol for traffic information systems, in: IEEE 66th Vehicular Technology Conference (VTC2007), September 2007, pp. 1947-1951.

[113] B. Williams, D. Mehta, T. Camp, W. Navidi, Predictive modeling of network wide broadcasting protocols for mobile ad hoc networks, IEEE Trans. Mobile Comput. 3 (5) (2004) 295-303.

[114] N. Wisitpongphan, O.K. Tonguz, Scalable broadcast strategies for ad hoc routing protocols, in: 1st International Symposium on Wireless Pervasive Computing, 2006, pp. 1-6.

[115] N. Wisitpongphan, O.K. Tonguz, J.S. Parikh, P. Mudalige, F. Bai, V. Sadekar, Broadcast storm mitigation techniques in vehicular ad hoc networks, IEEE Wirel. Commun. 14 (6) (2007) 84-94.

[116] C. Wu, S. Ohzahata, T. Kato, A low latency path diversity mechanism for sender-oriented broadcast protocols in vanets, Ac Hoc Netw. 11 (2013) 2059-2068.

[117] F. Xue, P.R. Kumar, The number of neighbors needed for connectivity of wireless networks, Wireless Netw. 10 (2004) 169181.

[118] M.B. Yassein, M.B. Khalaf, A. Al-Dubai, A new probabilistic broadcasting scheme for mobile ad hoc on-demand distance vector (aodv) routed networks, J. Supercomput. 53 (2010) 196-211.

[119] M.B. Yassein, M.O. Khaoua, L.M. Mackenzie, S. Papanastasiou, A Jamal, Improving route discovery in on-demand routing protocols using local topology information in manets, in: Proceedings of the ACM International Workshop on Performance Monitoring, Measurement, and Evaluation of Heterogeneous Wireless and Wired Networks (PM2HW2N '06), 2006, pp. 95-99.

[120] J. Yeo, D. Kotz, T. Henderson, Crawdad: a community resource for archiving wireless data at dartmouth, in: ACM SIGCOMM Computer Communication Review, 2008, pp. 21-22.

[121] J. Yick, B. Mukherjee, D. Ghosal, Wireless sensor networks survey, Comput. Networks 52 (2008) 2292-2330.

[122] J. Yoo, S. Choi, C. Kim, Control overhead reduction for neighbour knowledge acquisition in mobile ad hoc networks, Electron. Lett. 39 (2003) 740-741.

[123] J. Yoon, M. Liu, B. Noble, Random waypoint considered harmful, in Twenty-Second Annual Joint Conference of the IEEE Computer and Communications (INFOCOM 2003), vol. 2, 2003, pp. 1312-1321.

[124] X. Zeng, R. Bagrodia, M. Gerla, Glomosim: a library for paralle simulation of large-scale wireless networks, in: Proceedings of the 12th Workshop on Parallel and Distributed Simulations (PADS'98), 1998.

[125] H. Zhang, Z. Jiang, Performance analysis of broadcasting schemes in mobile ad hoc networks, IEEE Commun. Lett. 8 (12) (2004) 718 720.

[126] H. Zhang, Z. Jiang, Modeling and performance analysis of ad hoc broadcasting schemes, Perform. Eval. 63 (2006) 1196-1215.

[127] Q. Zhang, D.P. Agrawal, Analysis of leveled probabilistic routing in mobile ad hoc networks, in: IEEE International Conference on Communications, vol. 7, 2004, pp. 3896-3900.

[128] Q. Zhang, P. Agrawal, Dynamic probabilistic broadcasting in manets, J. Parallel Distrib. Comput. 65 (2005) 220-233.

[129] X.M. Zhang, E.B. Wang, J.J. Xia, D.K. Sung, A neighbor coverage based probabilistic rebroadcast for reducing routing overhead in mobile ad hoc networks, IEEE Trans. Mobile Comput. 11 (2012) 110.

[130] L. Zhou, G. Cui, H. Liu, Z. Wu, D. Luo, Nppb: a broadcast scheme in dense vanets, Inf. Technol. J. (2010) 1-10.

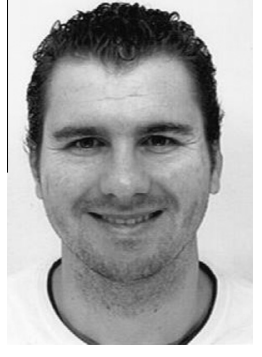

Daniel Gutiérrez Reina was born in Seville Spain, in 1983. He received the B.E. degree in electronic engineering and M.S. degree in electronics and telecommunications from the University of Seville, Seville, Spain, in 2009 and 2011 respectively, where he is currently working toward the Ph.D. degree. His current research interests include broadcast and routing protocols for wireless ad hoc networks.

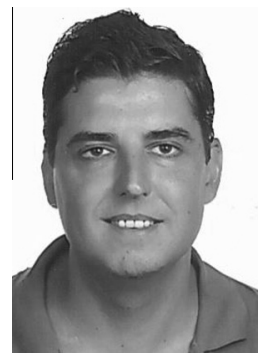

Sergio Toral was born in Rabat, Morocco, in 1972. He received the M.S. and Ph.D. degrees in electrical and electronic engineering from the University of Seville, Spain, in 1995 and 1999, respectively. He is currently a full Professor with the Department of Electronic Engineering, US. His main research interests include ad hoc networks and their routing protocols, deployment of wireless sensor networks, real-time and distributed systems, intelligent transportation systems, and embedded operating systems.

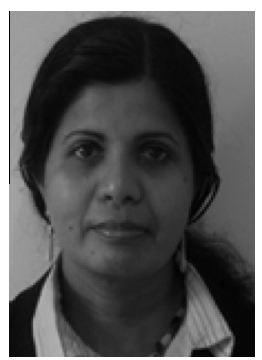

Princy Johnson gained a Bachelor of Engineering in Electronics and Communication Engineering from GCT, Coimbatore, India followed by a Master of Engineering in Applied Electronics from Guindy Engineering College, Madras, India, both with distinction. Funded through Commonwealth Scholarship she received her Ph.D. from King's College London in 2000. She has worked as a Research and Development engineer at Nortel Networks UK Ltd for two years during which she had a patent issued for a novel recon-gurable OADM. Dr. Johnson is at Liverpool John Moores University and her research interests include energy-effcient techniques and protocols for mobile wireless and sensor networks. She is currently developing an active EU research consortium on this topic.

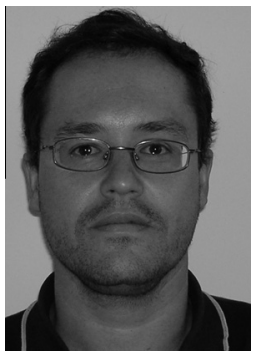

Federico Barrero received the M.Sc. and Ph.D. degrees in Electrical and Electronic Engineering from the University of Seville, Spain, in 1992 and 1998, respectively. In 1992, he joined the Electronic Engineering Department at the University of Seville, where he is currently an Associate Professor. His recent interests include sensor networks and control of multiphase AC drives. 